\title{
Adaptive Integral-Based Robust Q-S Synchronization and Parameter Identification of Nonlinear Hyperchaotic Complex Systems
}

\author{
Sami Ud Din (iD, ${ }^{1}$ Muhammad Rafiq Mufti ${ }^{D},{ }^{2}$ Humaira Afzal $\left(\mathbb{D},{ }^{3}\right.$ Majid Ali $\left(\mathbb{D},{ }^{1}\right.$ \\ and Muhammad Abdul Moiz $\mathrm{Zia}^{4}$ \\ ${ }^{1}$ Department of Electrical Engineering, NAMAL Institute Mianwali, Mianwali 42250, Pakistan \\ ${ }^{2}$ Department of Computer Science, COMSATS University Islamabad, Vehari Campus, Vehari 61100, Pakistan \\ ${ }^{3}$ Department of Computer Science, Bahauddin Zakariya University, Multan 60800, Pakistan \\ ${ }^{4}$ Department of Computer Science, University of Central Punjab, Lahore 54000, Pakistan
}

Correspondence should be addressed to Sami Ud Din; sami.uddin@namal.edu.pk

Received 20 December 2020; Revised 24 March 2021; Accepted 8 April 2021; Published 30 April 2021

Academic Editor: Xianggui Guo

Copyright $\odot 2021$ Sami Ud Din et al. This is an open access article distributed under the Creative Commons Attribution License, which permits unrestricted use, distribution, and reproduction in any medium, provided the original work is properly cited.

\begin{abstract}
This communique presents the Q-S synchronization of two nonidentical complex nonlinear hyperchaotic systems with unknown parameters. An adaptive controller based on adaptive integral sliding mode control and parameter update laws are designed to realize the synchronization and parameter identification to a given map vector. The aforementioned strategy's employment demands the transformation of a system into a specific structure containing a nominal part and some unknown terms (later on, these unknown terms will be computed adaptively). An integral sliding mode controller is used to stabilize the error system by designing nominal control accompanied by compensator control. For chattering suppression, a continuous compensator of smooth nature is used instead of conventional control. The stability of the proposed algorithm is established in an impressive way, using Lyapunov criteria. A numerical simulation is performed to illustrate the validity of the proposed synchronization scheme.
\end{abstract}

\section{Introduction}

Synchronization is considered to be a fundamental phenomenon that enables coherent behavior in the coupled systems. Chaos synchronization has been of great interest to researchers in recent years. The hyperchaotic systems are very sensitive towards initial conditions and possess at least two positive Lyapunov exponents. They have bounded trajectories in the phase space, exhibit more complex nonlinear behavior, and so on. Hyperchaos was first formalized in 1979 by Rossler [1]. Many other hyperchaotic systems were reported later on [2-4]. In [5], the Wien-bridge coupled oscillator system was also indicated to be hyperchaotic. In 1990, Pecora and Carroll [6] introduced the synchronization of two identical chaotic systems with different initial conditions. The field of chaotic synchronization flourished extensively in the last two decades, and many new strategies are also proposed in this regard, including complete synchronization $[7,8]$, lag synchronization $[9,10]$, inverse lag synchronization [11], inverse $\pi$-lag synchronization [12], generalized synchronization [13-15], multiple chaotic systems' synchronization [16, 17], phase synchronization [18], antisynchronization [19, 20], partial synchronization [21, 22], Q-S synchronization [23, 24], projective synchronization $[25,26]$, and fractional chaos synchronization $[27,28]$. In recent years, generalized synchronization (GS) of chaotic systems was widely investigated. The researchers in the control community are still exploring the new dimensions of inverse generalized synchronization (IGS), which is also considered an attractive idea of this era regarding secure communication.

The sliding mode control (SMC) has attracted researchers' attention in recent years due to its robust response to model insensitivity towards nonlinearities, ease of 
implementation, low computational cost, and simplicity in feedback design [29-34]. Moreover, SMC is independent of external interferences and only depends on the switching surface design (also called the sliding surface) [31]. Usually, the strategy of SMC works in two phases, namely, the reaching phase and the sliding phase. During the first phase (reaching phase), the system has no insensitivity property [31], and system gain is designed to guide the system trajectory towards the sliding surface. In contrast, during the second (sliding) phase, the system is forced to slide on the 'sliding surface.' During this phase, the system remains insensitive to external disturbances and parametric variations. The only problem that lies in SMC is the chattering (high-frequency oscillation) phenomenon, which is significantly suppressed by using the integral sliding mode control (ISMC) [30, 31]. ISMC is also robust in the whole state space due to the absence of the reaching phase [30,31]; therefore, it guarantees robustness from the very beginning at the initial time instance. ISMC is employed in combination with nominal control accompanied with discontinuous control that eliminates the parameter uncertainties and nonsmooth nonlinearities from the system model $[30,31]$. The beauty of ISMC can be further enhanced in terms of system performance and become more productive if parameters used in the system/controller may be computed adaptively. Specifically considering hyperchaotic systems, Rikitake and Lorenz system always remains in the spotlight due to its complicated dynamical response and vital relevance in engineering $[18,19]$. In this regard, the authors have posed adaptive integral sliding mode control (AISMC) for complex hyperchaotic systems. Our contributions to this paper are as follows:

(i) The contribution is presented in a way that authors have proposed the Q-S synchronization for $n$-dimensional chaotic systems.

(ii) The proposed methodology is based on the adaptive integral sliding mode control (AISMC), which is relatively more robust than other controllers proposed in the literature. The aforementioned algorithm is also capable of managing matched uncertainties. Moreover, it will also be beneficial in terms of system performance and robustness enhancement.

(iii) The system's stability based on the aforementioned algorithms is also established in an impressive way using Lyapunov criteria.

(iv) Furthermore, to verify the effectiveness of the proposed method, we apply it to $4 \mathrm{D}$ continuoustime chaotic systems.

The rest of the paper is organized as follows. The design and definition of Q-S synchronization of nonidentical systems are given in Section 2. Q-S synchronization and parameter identification of two different systems with the same orders are theoretically investigated in Section 3. A numerical-based illustrative example is presented in Section 4, and conclusions are drawn in Section 5.

\section{Q-S Synchronization}

The system shown in (1) is a nonidentical drive system, while the response system is presented by equation (2) with fully unknown parameters as follows:

$$
\begin{aligned}
& \dot{x}=f(x)+F(x) \theta, \\
& \dot{y}=g(y)+G(y) \vartheta+u(x, y),
\end{aligned}
$$

where $x=\left(x_{1}, x_{2}, \ldots, x_{n}\right)^{T}$ and $y=\left(y_{1}, y_{2}, \ldots, y_{n}\right)^{T}$ are the vectors presenting the states of drive (1) and response system (2), respectively, $\theta \in \mathfrak{R}^{p}$ and $\vartheta \in \mathfrak{R}^{q}$ are real vectors with parameters (unknown), $F(x) \in \Re^{n \times p}$ and $G(y) \in \Re^{n \times q}$ are real matrices, $f(x) \in \mathfrak{R}^{n}$ and $g(y) \in \mathfrak{R}^{n}$ are vectors of nonlinear functions, and $u(x, y) \in \mathfrak{R}^{n}$ is the control vector.

Definition 1. For the system shown in (1) and (2), Q-S synchronization is attained if a controller $u(x, y)$ and two given maps $\phi(x) \in \mathfrak{R}^{n} \longrightarrow \mathfrak{R}^{n}$ and $\psi(y) \in \mathfrak{R}^{n} \longrightarrow \mathfrak{R}^{n}$ exist such that

$$
\lim _{\mathrm{t} \longrightarrow \infty}\|\psi(y)-\phi(x)\|=0,
$$

where $\|$.$\| represents the matrix norm.$

$$
\phi(x)=\left[\phi_{1}(x) \phi_{2}(x) \ldots \phi_{n}(x)\right]^{T} \text { and } \psi(y)=\left[\psi_{1}(y) \psi_{2}(y) \ldots \psi_{n}(y)\right]^{T} \text {, }
$$

are nonzero vector maps whose elements are continuously differentiable functions of $x$ and $y$, respectively.

\section{General Scheme of Q-S Synchronization and Parameter Identification}

Q-S synchronization error vectors are defined as

$$
e=\psi(y)-\phi(x) \text {, }
$$

where $e=\left(e_{1}, e_{2}, \ldots, e_{n}\right)^{T} \in \mathfrak{R}^{n}$. Let $J_{x}=\partial / \partial x \phi(x)$ and $J_{y}=\partial / \partial y \psi(y)$ be Jacobian of the mappings $\phi(x)$ and $\psi(y)$, respectively. Choose $\psi(y)$ such that the inverse of $J_{y}$ exists. Consider $\widehat{\theta}$ and $\widehat{\vartheta}$ are the estimates of $\theta$ and $\vartheta$, respectively, and also assume $\widetilde{\theta}=\theta-\widehat{\theta}$ and $\widetilde{\vartheta}=\widehat{\vartheta}-\vartheta$ are the parametric estimation errors of $\theta$ and $\vartheta$, respectively. Differentiating equation (5) with respect to time, the Q-S synchronization error dynamical system is obtained as 


$$
\begin{aligned}
\dot{e} & =\dot{\psi}(y)-\dot{\phi}(x)=\frac{\partial}{\partial y}\{\psi(y)\} \dot{y}-\frac{\partial}{\partial x}\{\phi(x)\} \dot{x}=J_{y} \dot{y}-J_{x} \dot{x} \\
& =J_{y}\{g(y)+G(y) \widehat{\vartheta}+G(y) \widetilde{\vartheta}+u(x, y)\}-J_{x}\{f(x)+F(x) \widehat{\theta}+F(x) \widetilde{\theta}\} \\
& =J_{y}\{g(y)+G(y) \widehat{\vartheta}\}+J_{y} u(x, y)-J_{x}\{f(x)+F(x) \hat{\theta}\}+J_{y} G(y) \widetilde{\vartheta}-J_{x} F(x) \widetilde{\theta},
\end{aligned}
$$

by choosing

$$
u(x, y)=J_{y}^{-1}\left[-J_{y}\{g(y)+G(y) \hat{\vartheta}\}-J_{x}\{f(x)+F(x) \hat{\theta}\}+e e\right],
$$

where $e e=\left[e_{2} e_{3} \ldots e_{n} v\right]^{T}$ and $v$ is the new input. Then, error system (6) becomes

$$
\dot{e}=e e+J_{y} G(y) \widetilde{\vartheta}-J_{x} F(x) \widetilde{\theta},
$$

or

$$
\left[\begin{array}{c}
\dot{e}_{1} \\
\dot{e}_{2} \\
\vdots \\
\dot{e}_{n-1} \\
\dot{e}_{n}
\end{array}\right]=\left[\begin{array}{c}
e_{2} \\
e_{3} \\
\vdots \\
e_{n} \\
v
\end{array}\right]+J_{y} G(y) \widetilde{\vartheta}-J_{x} F(x) \tilde{\theta} .
$$

Choosing the nominal system for (9), we aim to employ the ISMC:

$$
\left[\begin{array}{c}
\dot{e}_{1} \\
\dot{e}_{2} \\
\vdots \\
\dot{e}_{n-1} \\
\dot{e}_{n}
\end{array}\right]=\left[\begin{array}{c}
e_{2} \\
e_{3} \\
\vdots \\
e_{n} \\
v_{0}
\end{array}\right]
$$

Switching surfaces for the nominal system shown in (9) are displayed as follows:

$$
\sigma_{0}=C e=e_{1}+\sum_{k=2}^{n-1} c_{k} e_{k}+e_{n}
$$

where $C=\left[1 c_{2} \ldots c_{n} 1\right]$ is selected such that $\sigma_{0}$ gets to be the Hurwitz polynomial. Then,

$$
\dot{\sigma}_{0}=C \dot{e}=e_{2}+\sum_{k=2}^{n-1} c_{k} e_{k+1}+v_{0} .
$$

By choosing $v_{0}=-e_{2}-\sum_{k=2}^{n-1} c_{k} e_{(k+1)}-k \sigma_{0}$, where $k>0$, we have $\dot{\sigma}_{0}=-k \sigma_{0}$; therefore, the system shown in (10) is stable asymptotically.

Now, the switching surface for the system shown in (9) is considered as $\sigma=\sigma_{0}+z$, and $z$ is an integral term (which will be computed later). To skip the reaching phase and for the establishment of the sliding phase at the very instant, select $z(0)$ such that $\sigma(0)=0$. This condition guarantees the establishment of a sliding phase at the start by skipping the reaching phase. Choose $v=v_{0}+v_{s}$ where the nominal input is shown as $v_{0}$ and $v_{s}$ which represent compensator terms. Then,

$$
\begin{aligned}
\dot{\sigma}= & \dot{\sigma}_{0}+\dot{z}=C \dot{e}+\dot{z}=e_{2}+\sum_{k=2}^{n-1} c_{k} e_{(k+1)} \\
& +v_{0}+v_{s}+\dot{z}+C J_{y} G(y) \widetilde{\vartheta}-C J_{x} F(x) \tilde{\theta} .
\end{aligned}
$$

By choosing a Lyapunov function $V=1 / 2 \sigma^{2}+1 / 2 \widetilde{\theta}^{T} \widetilde{\theta}+$ $1 / 2 \widetilde{\vartheta}^{T} \widetilde{\vartheta}$, adaptive laws are designed for $\widetilde{\theta}, \widehat{\theta}, \widetilde{\vartheta}$, and $\widehat{\vartheta}$, and take into account $v_{s}$ so that $\dot{V}<0$.

Theorem 1. The Lyapunov function is considered as

$$
V=1 / 2 \sigma^{2}+1 / 2 \tilde{\theta}+1 / 2 \widetilde{\vartheta}^{T} \widetilde{\vartheta} .
$$

Moreover, the adaptive laws are chosen as

$$
\begin{array}{lll}
\dot{z}=-e_{2}-\sum_{k=2}^{n-1} c_{k} e_{(k+1)}-v_{0}, & v_{s}=-k \sigma, \\
\dot{\tilde{\vartheta}}=-\sigma G^{T}(y) J^{T} C^{T}-k_{2} \widetilde{\vartheta}, & \dot{\tilde{\vartheta}}=-\dot{\tilde{\vartheta}}, & \dot{\tilde{\theta}}=-\dot{\tilde{\theta}}, \\
\dot{\tilde{\theta}}=-\sigma F^{T}(x) C^{T}-k_{1} \tilde{\theta}, & \dot{\tilde{\theta}}=-\dot{\tilde{\theta}}, & \dot{\bar{\vartheta}}=-\dot{\tilde{\vartheta}},
\end{array}
$$

for $\tilde{\theta}, \widehat{\theta}, \widetilde{\vartheta}$, and $\widehat{\vartheta}$, and $v_{s}$ results in $\dot{V}<0$ if $k_{1}, k_{2}>0$.

Proof. As

$$
\begin{aligned}
\dot{V} & =\sigma \dot{\sigma}+\tilde{\theta}^{T} \dot{\tilde{\theta}}+\tilde{\vartheta}^{T} \dot{\tilde{\vartheta}}=\sigma\left\{e_{2}+\sum_{k=2}^{n-1} c_{k} e_{(k+1)}+v_{0}+v_{s}+\dot{z}+C J_{y} G(y) \tilde{\vartheta}-C J_{x} F(x) \tilde{\theta}\right\}+\tilde{\theta}^{T} \dot{\tilde{\theta}}+\widetilde{\vartheta}^{T} \dot{\tilde{\vartheta}} \\
& =\sigma\left\{e_{2}+\sum_{k=2}^{n-1} c_{k} e_{(k+1)}+v_{0}+v_{s}+\dot{z}\right\}+\tilde{\theta}^{T}\left\{\dot{\tilde{\theta}}-F^{T}(x) J_{x}^{T} C^{T}\right\}+\tilde{\vartheta}^{T}\left\{\dot{\tilde{\vartheta}}+G^{T}(y) J_{y}^{T} C^{T}\right\},
\end{aligned}
$$


by using

$$
\begin{array}{lll}
\dot{z}=-e_{2}-\sum_{k=2}^{n-1} c_{k} e_{(k+1)}-v_{0}, & v_{s}=-k \sigma, \\
\dot{\tilde{\theta}}=\sigma F^{T}(x) J_{x}^{T} C^{T}-k_{1} \tilde{\theta}, & \dot{\tilde{\theta}}=-\dot{\tilde{\theta}}, & \dot{\hat{\theta}}=-\dot{\widetilde{\theta}}, \\
\dot{\tilde{\vartheta}}=-\sigma G^{T}(y) J_{y}^{T} C^{T}-k_{2} \widetilde{\vartheta}, & \dot{\tilde{\vartheta}}=-\dot{\widetilde{\vartheta}}, \quad \dot{\vec{\vartheta}}=-\dot{\widetilde{\vartheta}}, \\
\dot{V}=-k \sigma^{2}-k_{1} \tilde{\theta}^{T} \tilde{\theta}-k_{2} \widetilde{\vartheta}^{T} \widetilde{\vartheta} . &
\end{array}
$$

From the above equation, we may result in $\sigma, \widetilde{\theta}, \widetilde{\vartheta} \longrightarrow 0$. As $\sigma$ is approaching 0 , accordingly, $e \longrightarrow 0$.

\section{Example}

Q-S-based synchronization of two hyperchaotic nonidentical nonlinear complex systems along with the same order is investigated here. The hyperchaotic Lorenz-type system proposed in [35] is displayed as follows:

$$
\begin{aligned}
& \dot{x}_{1}=a_{1}\left(x_{2}-x_{1}\right), \\
& \left\{\begin{array}{l}
\dot{x}_{2}=a_{2} x_{1}-x_{1} x_{3}-a_{3} x_{2}+x_{4}, \\
\dot{x}_{3}=x_{1} x_{2}-a_{4} x_{3}, \\
\dot{x}_{4}=-a_{5} x_{2}-a_{6} x_{4},
\end{array}\right.
\end{aligned}
$$

where $x_{1}, x_{2}, x_{3}$, and $x_{4}$ are state variables and $a_{1}, a_{2}, a_{3}, a_{4}, a_{5}$, and $a_{6}$ are system parameters (with positive constant values). When $a_{1}=12, a_{2}=23$, $a_{3}=1, a_{4}=2.1, a_{5}=6$, and $a_{6}=0.2$ and $x(0)=[1,2,3,4]^{T}$, system (19) is hyperchaotic, and its attractor is shown in Figures 1 and 2. A four-wing hyperchaotic system is reported in $[35,36]$ and has one unstable equilibrium.

$$
\begin{aligned}
& \dot{y}_{1}=b_{1} y_{1}-y_{2} y_{3}+y_{4}+u_{1}, \\
& \left\{\begin{array}{l}
\dot{y}_{2}=y_{1} y_{3}-b_{2} y_{2}+u_{2}, \\
\dot{y}_{3}=y_{1} y_{2}-b_{3} y_{3}+y_{1} y_{4}+u_{3}, \\
\dot{y}_{4}=-y_{2}+u_{4} .
\end{array}\right.
\end{aligned}
$$

where $y_{1}, y_{2}, y_{3}$, and $y_{4}$ are state variables, $b_{1}, b_{2}$, and $b_{3}$ are positive constant system parameters, and $u_{1}, u_{2}, u_{3}$, and $u_{4}$ are controller inputs. When $b_{1}=8, b_{2}=40$, and $b_{3}=14.9$ and $y(0)=[10,1,10,1]^{T}$, the system shown in (20) stays in the chaotic orbits without control, which can be seen in Figures 3 and 4 .

Let $\widehat{a}_{i}, \widehat{b}_{k}, i=1, \ldots, 4 ; k=1$ to 3 , be the estimates of $a_{i}, b_{k}, i=1, \ldots, 4 ; k=1$ to 3 , along with $\tilde{a}_{i}=a_{i}-\widehat{a}_{i}$, $i=1, \ldots, 4$, and $\widetilde{b}_{k}=b_{k}-\widehat{b}_{k}, k=1$ to 3 , which represent the errors in the estimations of $\tilde{a}_{i}, \widetilde{b}_{k}, i=1, \ldots, 4 ; k=1$ to 3 , respectively.

Then, systems (19) and (20) can be written as

$$
\left\{\begin{array}{l}
\dot{x}_{1}=\widehat{a}_{1}\left(x_{2}-x_{1}\right)+\tilde{a}_{1}\left(x_{2}-x_{1}\right), \\
\dot{x}_{2}=\widehat{a}_{2} x_{1}+\widetilde{a}_{2} x_{1}-x_{1} x_{3}-\widehat{a}_{3} x_{2}-\widetilde{a}_{3} x_{2}+x_{4}, \\
\dot{x}_{3}=x_{1} x_{2}-\widehat{a}_{4} x_{3}-\tilde{a}_{4} x_{3}, \\
\dot{x}_{4}=-\widehat{a}_{5} x_{2}-\tilde{a}_{5} x_{2}-\widehat{a}_{6} x_{4}-\tilde{a}_{6} x_{4},
\end{array}\right.
$$

and

$$
\left\{\begin{array}{l}
\dot{y}_{1}=\widehat{b}_{1} y_{1}-\widetilde{b}_{1} y_{1}-y_{2} y_{3}+y_{4}+u_{1}, \\
\dot{y}_{2}=y_{1} y_{3}-\widehat{b}_{2} y_{2}-\widetilde{b}_{2} y_{2}+u_{2}, \\
\dot{y}_{3}=y_{1} y_{2}-\widehat{b}_{3} y_{3}-\widetilde{b}_{3} y_{3}+y_{1} y_{4}+u_{3}, \\
\dot{y}_{4}=-y_{2}+u_{4} .
\end{array}\right.
$$

Systems shown in (21) and (22) could be written as

$$
\begin{aligned}
& \dot{x}=f(x)+F(x) \widehat{\theta}+F(x) \tilde{\theta}, \\
& \dot{y}=g(y)+G(y) \widehat{\vartheta}+G(y) \widetilde{\vartheta}+u,
\end{aligned}
$$

where

$$
\begin{aligned}
& x=\left[\begin{array}{l}
x_{1} \\
x_{2} \\
x_{3} \\
x_{4}
\end{array}\right], f(x)=\left[\begin{array}{c}
0 \\
-x_{1} x_{3}+x_{4} \\
x_{1} x_{2} \\
0
\end{array}\right], F(x)=\left[\begin{array}{cccccc}
x_{2}-x_{1} & 0 & 0 & 0 & 0 & 0 \\
0 & x_{1} & -x_{2} & 0 & 0 & 0 \\
0 & 0 & 0 & -x_{3} & 0 & 0 \\
0 & 0 & 0 & 0 & -x_{2} & -x_{4}
\end{array}\right], y=\left[\begin{array}{l}
y_{1} \\
y_{2} \\
y_{3} \\
y_{4}
\end{array}\right], \\
& g(y)=\left[\begin{array}{c}
-y_{2} y_{3}+y_{4} \\
-y_{1} y_{3} \\
y_{1} y_{2}+y_{1} y_{4} \\
-y_{2}
\end{array}\right], u=\left[\begin{array}{c}
u_{1} \\
u_{2} \\
u_{3} \\
u_{4}
\end{array}\right], G(y)=\left[\begin{array}{ccc}
y_{1} & 0 & 0 \\
0 & -y_{2} & 0 \\
0 & 0 & -y_{3} \\
0 & 0 & 0
\end{array}\right], \widehat{\vartheta}=\left[\begin{array}{c}
\widehat{b}_{1} \\
\widehat{b}_{2} \\
\widehat{b}_{3}
\end{array}\right], \widehat{\vartheta}=\left[\begin{array}{c}
\widetilde{b}_{1} \\
\widetilde{b}_{2} \\
\widetilde{b}_{3}
\end{array}\right] \text {. } \\
& \phi(x)=\left[\begin{array}{llll}
x_{2}+3 x_{3} & 2 x_{1}-3 x_{4} & x_{3}-x_{1} & 3 x_{2}+x_{4}
\end{array}\right], \\
& \psi(y)=\left[\begin{array}{llll}
y_{1}+2 y_{4} & 2 y_{2}-3 y_{3} & y_{3}+y_{4} & 3 y_{2}+2 y_{4}
\end{array}\right] .
\end{aligned}
$$

The vector maps $\phi(x)$ and $\psi(y)$ are chosen as

Then, 


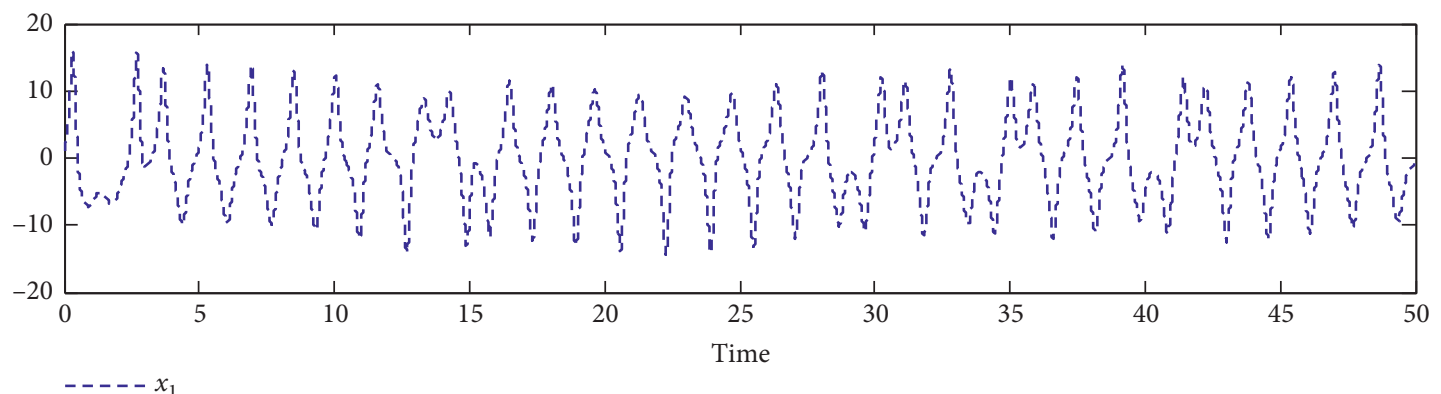

(a)

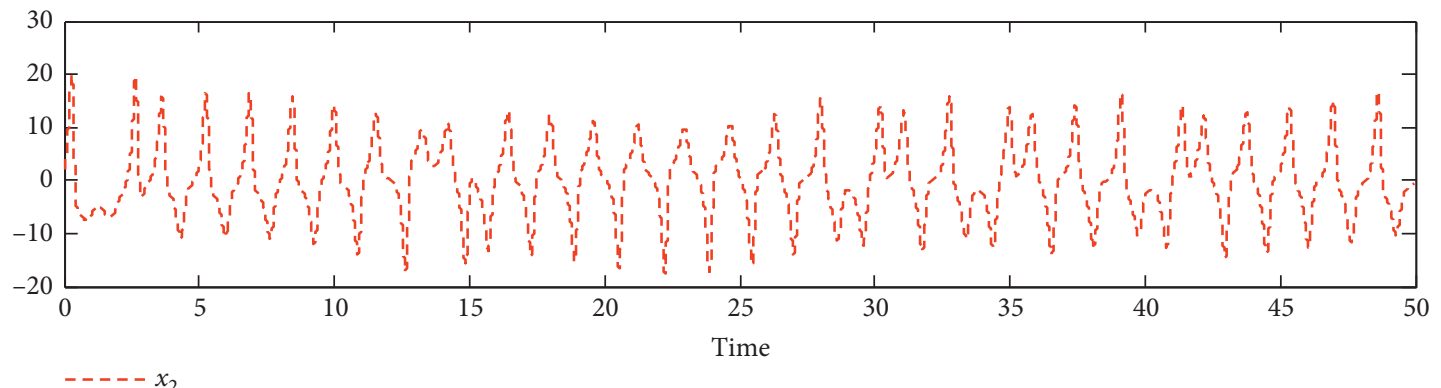

(b)

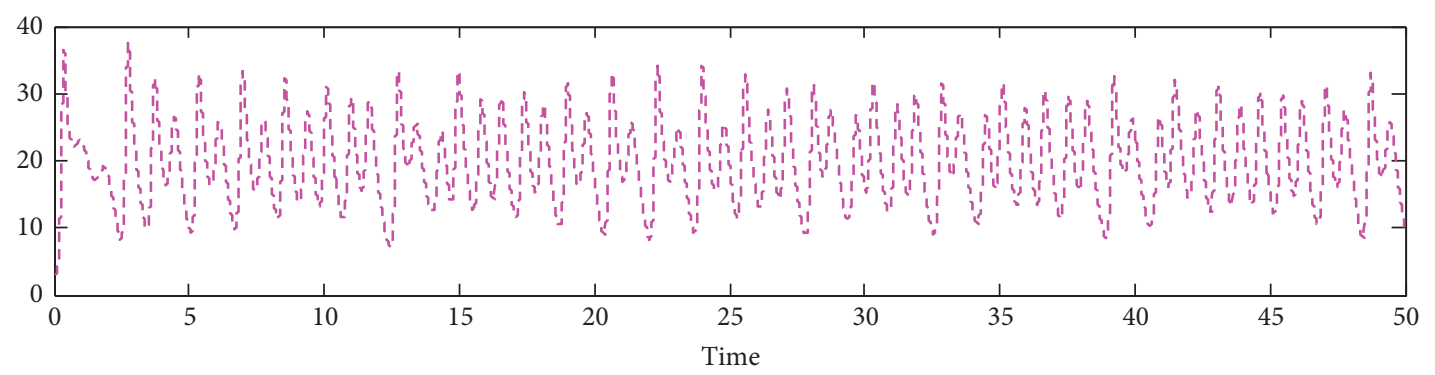

(c)

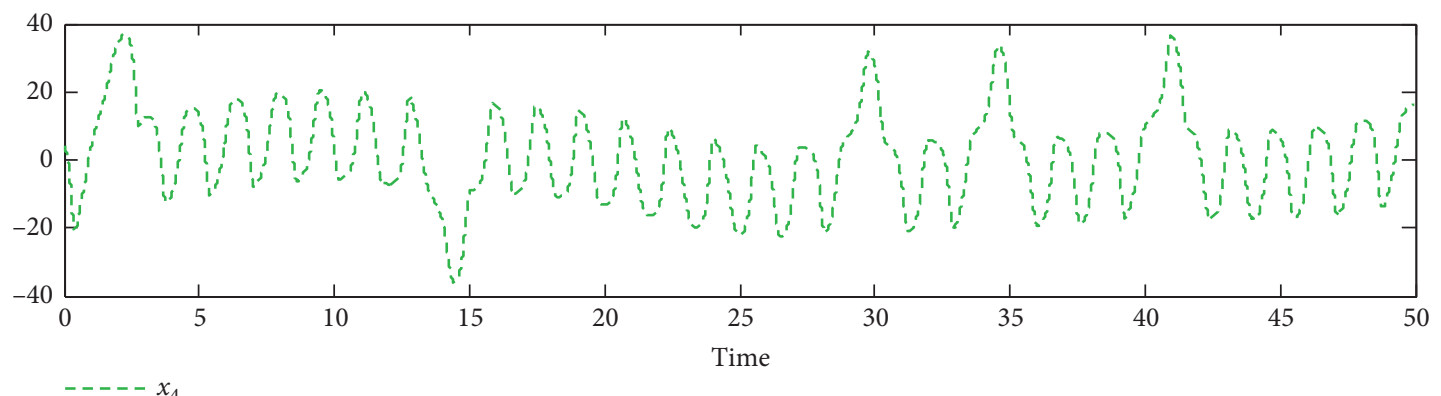

(d)

Figure 1: Continued. 


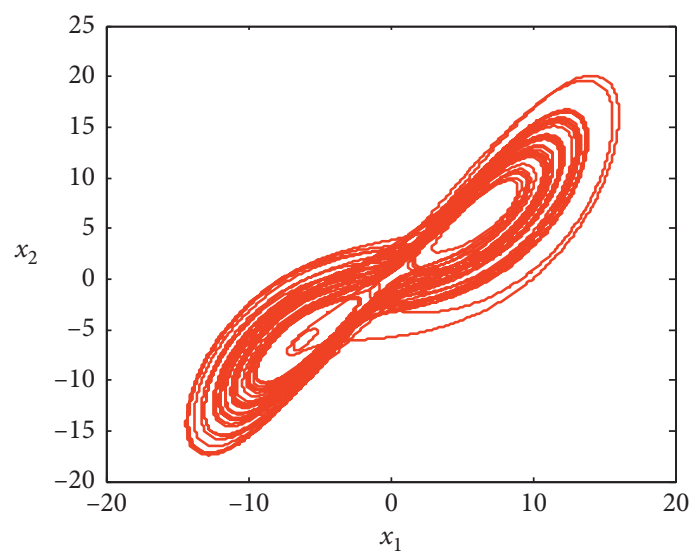

(e)

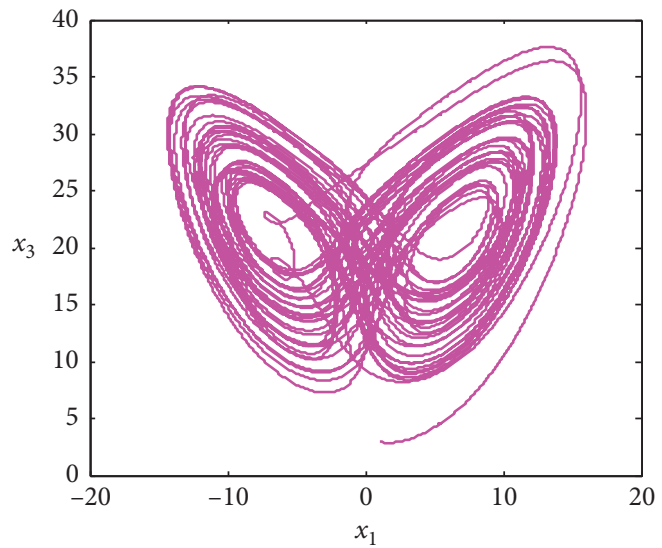

(f)

FIGURE 1: Graphical representation of state variables and the phase portrait (3D) of the Lorenz hyperchaotic system.

$$
J_{x}=\frac{\partial}{\partial x} \phi(x)=\left[\begin{array}{rrrr}
0 & 1 & 3 & 0 \\
2 & 0 & 0 & -3 \\
-1 & 0 & 1 & 0 \\
0 & 3 & 0 & 1
\end{array}\right], J_{y}=\frac{\partial}{\partial y} \psi(y)=\left[\begin{array}{cccc}
1 & 0 & 0 & 2 \\
0 & 2 & -3 & 0 \\
0 & 0 & 1 & 1 \\
0 & 3 & 0 & 2
\end{array}\right]
$$

The error is characterized as

$$
e=\psi(y)-\phi(x)
$$

i.e.,

$$
\left\{\begin{array}{l}
e_{1}=\psi_{1}-\phi_{1}=\left(y_{1}+2 y_{4}\right)-\left(x_{2}+3 x_{3}\right), \\
e_{2}=\psi_{2}-\phi_{2}=\left(2 y_{2}-3 y_{3}\right)-\left(2 x_{1}-3 x_{4}\right), \\
e_{3}=\psi_{3}-\phi_{3}=\left(y_{3}+y_{4}\right)-\left(x_{3}-x_{1}\right), \\
e_{4}=\psi_{4}-\phi_{4}=\left(3 y_{2}+2 y_{4}\right)-\left(3 x_{2}+x_{4}\right) .
\end{array}\right.
$$

Now, the dynamical error system becomes

$$
\dot{e}=J_{y} \dot{y}-J_{x} \dot{x}=J_{y}\{g(y)+G(y) \widehat{\vartheta}+G(y) \tilde{\vartheta}+u\}-J_{x}\{f(x)+F(x) \hat{\theta}+F(x) \tilde{\theta}\}
$$




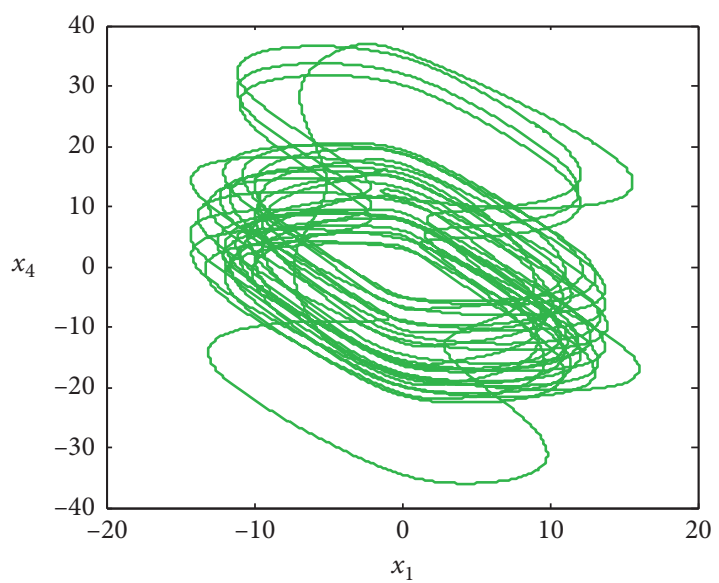

(a)

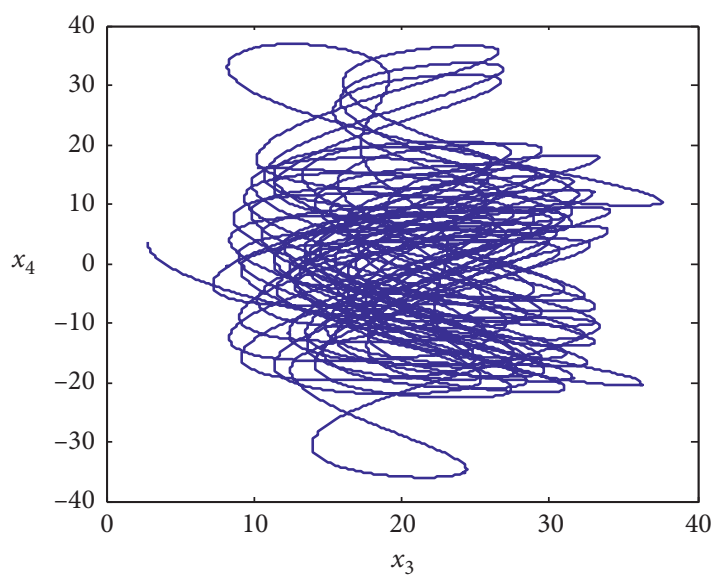

(c)

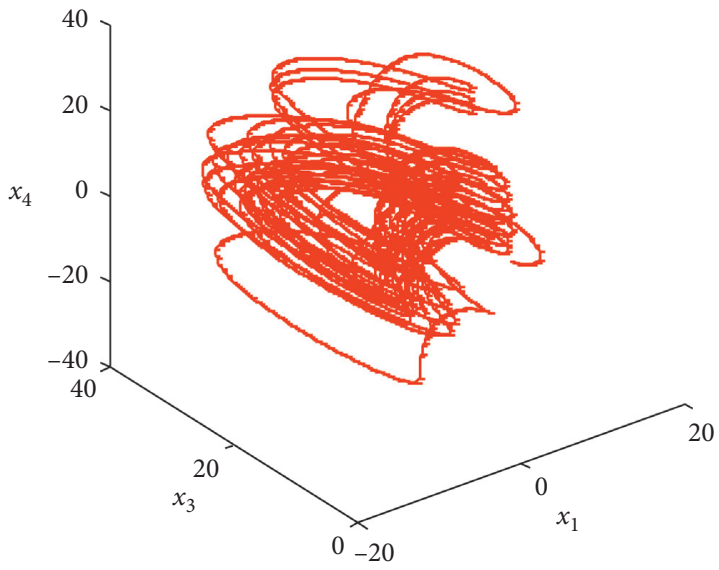

(e)

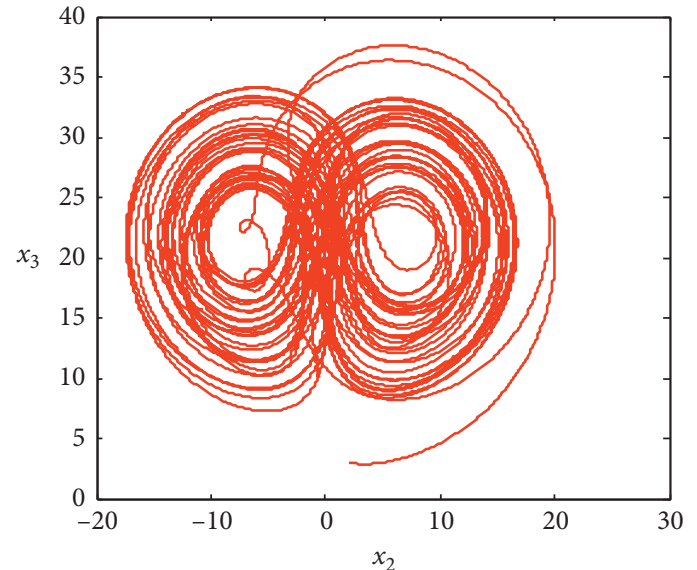

(b)

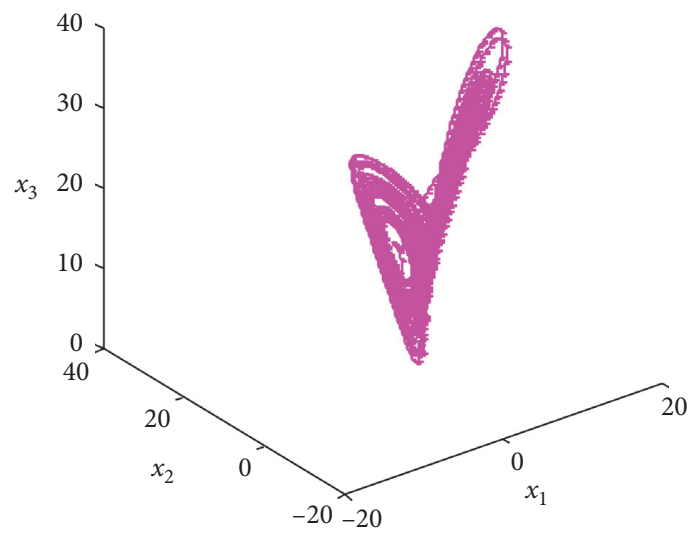

(d)

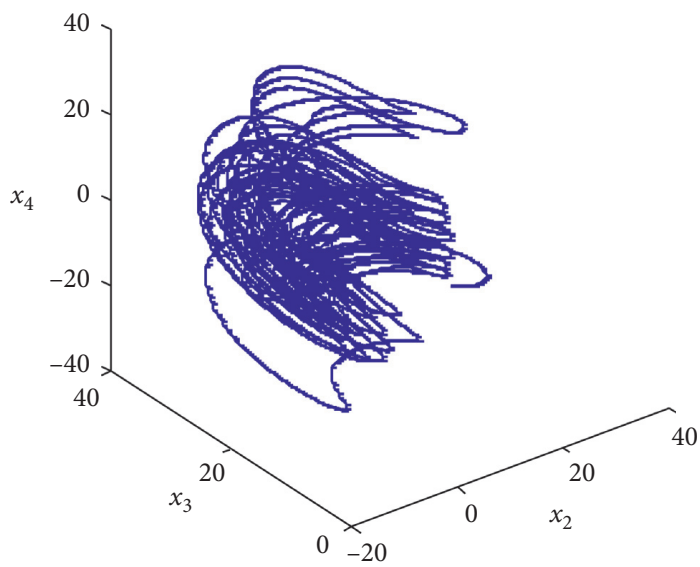

(f)

FIgURE 2: 3D phase portrait of the Lorenz nonlinear hyperchaotic complex system when $a_{1}=12, a_{2}=23, a_{3}=1, a_{4}=2.1, a_{5}=6$, and $a_{6}=0.2$ at $x(0)=[1,2,3,4]^{T}$. 


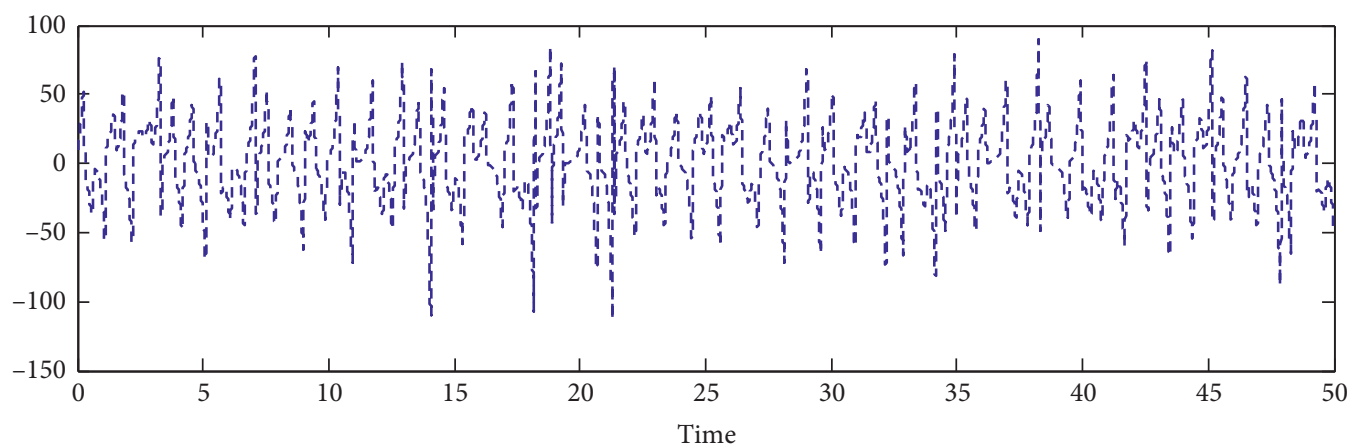

$---y_{1}$

(a)

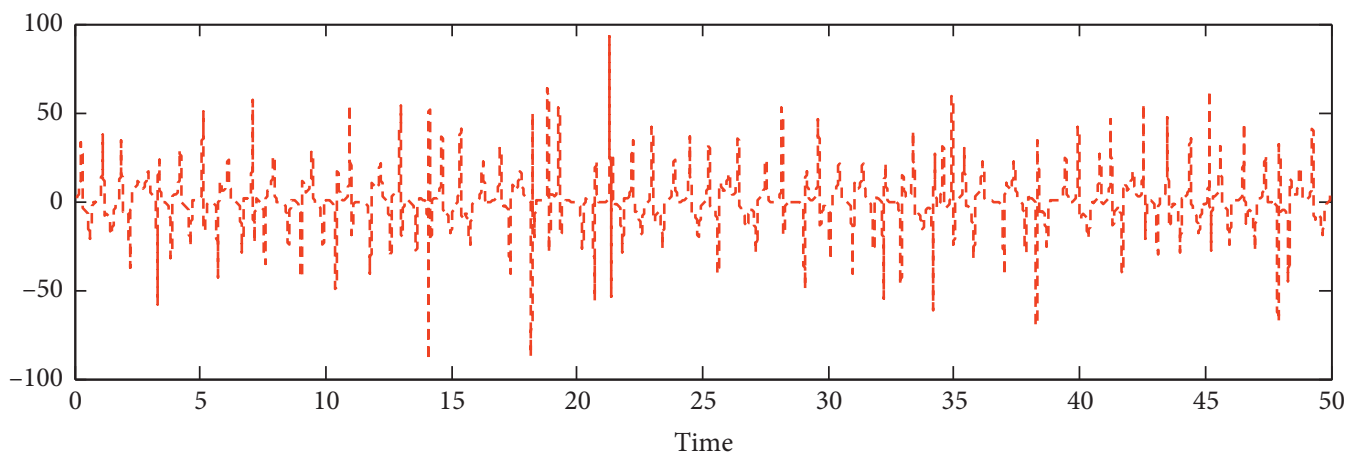

$---y_{2}$

(b)

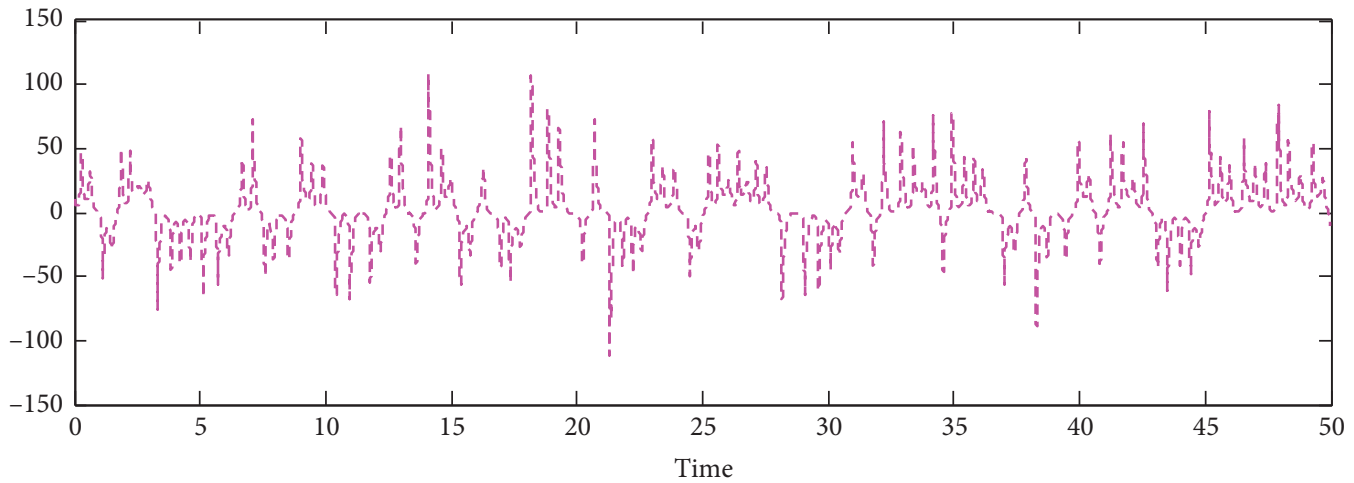

$---y_{3}$

(c)

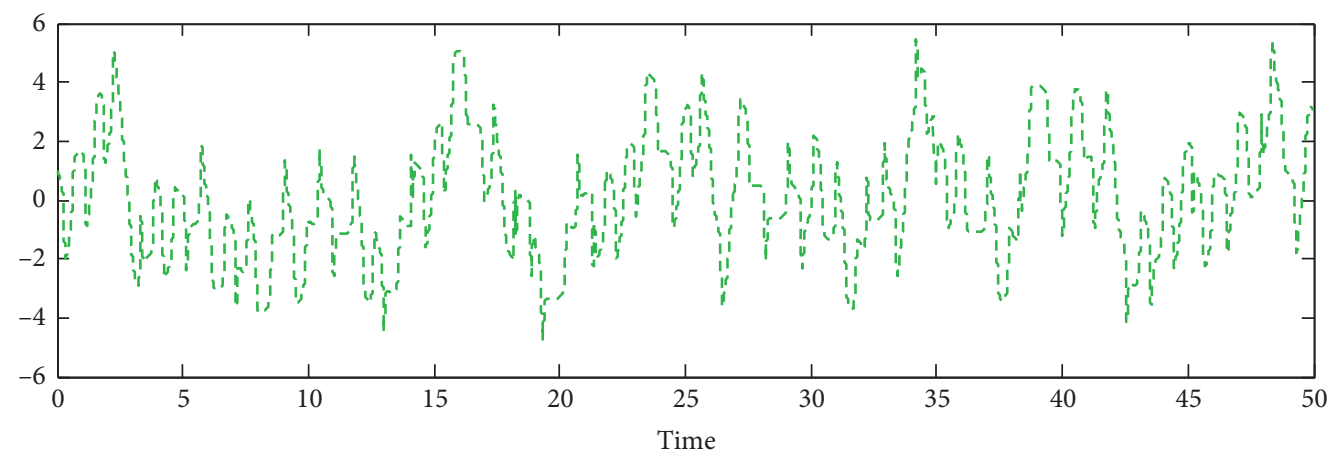

$---y_{4}$

(d)

Figure 3: Time history of state variables of the four-wing nonlinear hyperchaotic complex system. 


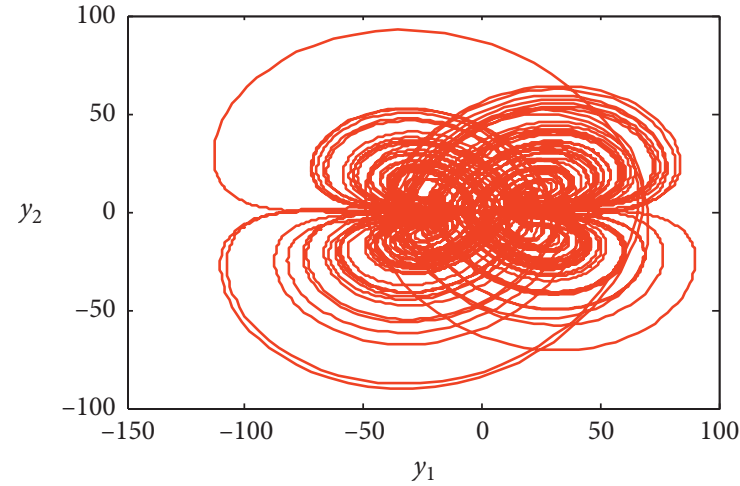

(a)

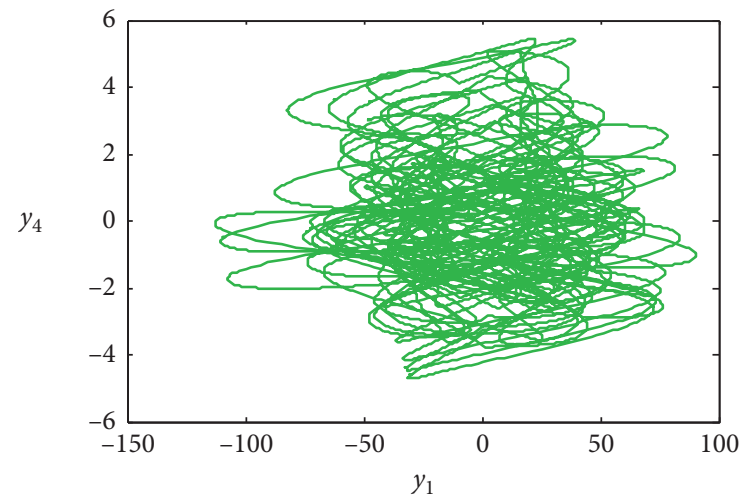

(c)

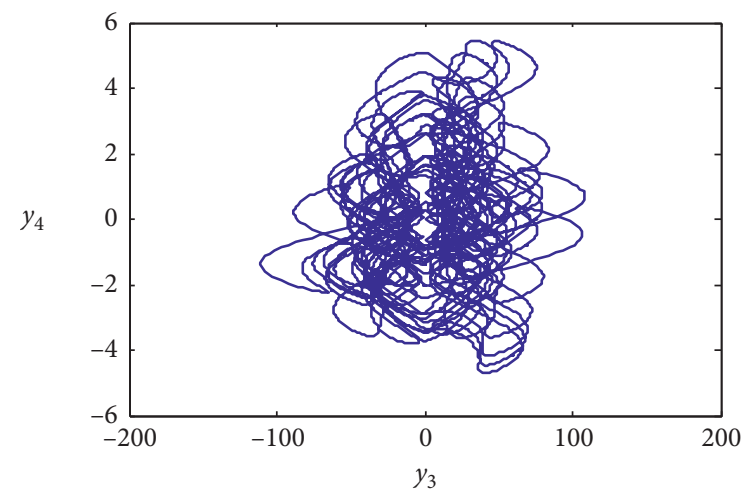

(e)

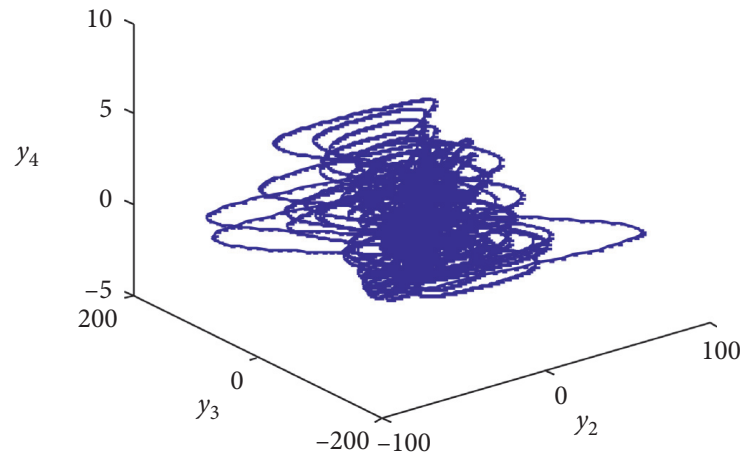

(g)

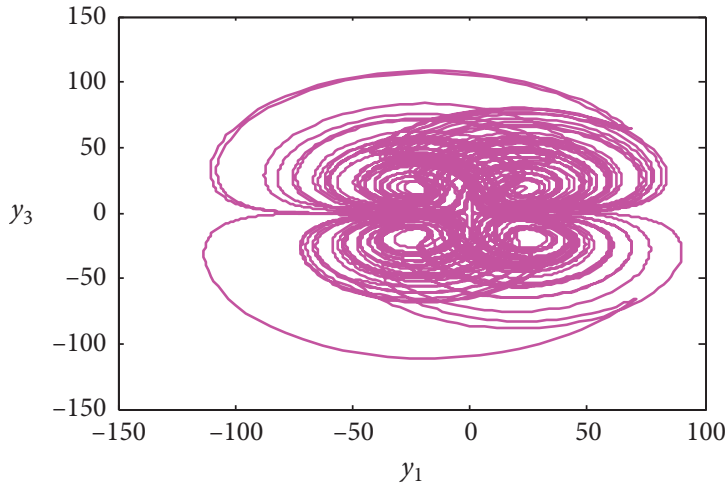

(b)

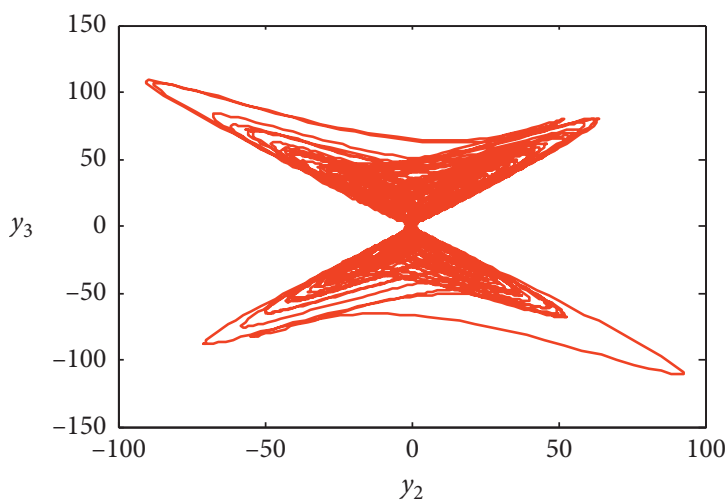

(d)

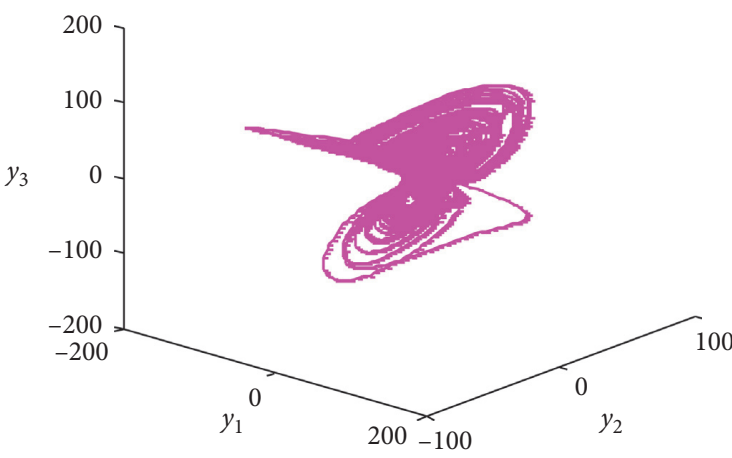

(f)

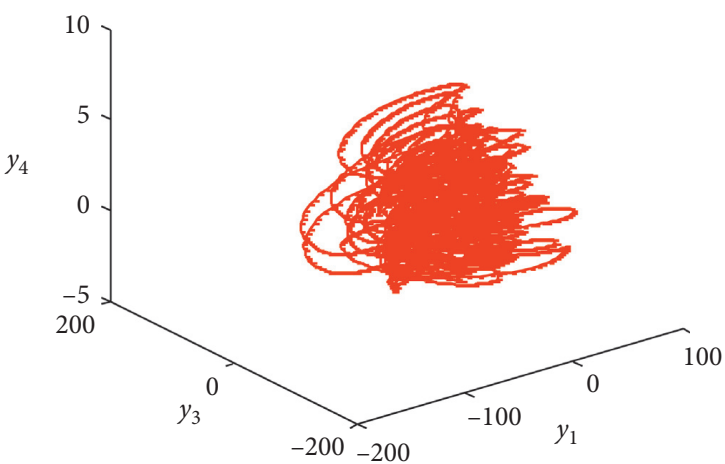

(h)

Figure 4: Phase portrait (3D) of the four-wing nonlinear hyperchaotic system when $b_{1}=8, b_{2}=40$, and $b_{3}=14.9$ and $y(0)=[10,1,10,1]^{T}$. 
or

$$
\begin{aligned}
& \dot{e}=J_{y}\{g(y)+G(y) \widehat{\vartheta}\}-J_{x}\{f(x)+F(x) \widehat{\theta}\}+J_{y} G(y) \widetilde{\vartheta}-J_{x} F(x) \widetilde{\theta}+J_{y} u \\
& =\left[\begin{array}{cccc}
1 & 0 & 0 & 2 \\
0 & 2 & -3 & 0 \\
0 & 0 & 1 & 1 \\
0 & 3 & 0 & 2
\end{array}\right]\left\{\left[\begin{array}{c}
-y_{2} y_{3}+y_{4} \\
-y_{1} y_{3} \\
y_{1} y_{2}+y_{1} y_{4} \\
-y_{2}
\end{array}\right]+\left[\begin{array}{c}
y_{1} \widehat{b}_{1} \\
y_{2} \widehat{b}_{2} \\
y_{3} \widehat{b}_{3} \\
0
\end{array}\right]\right\}-\left[\begin{array}{cccc}
0 & 1 & 3 & 0 \\
2 & 0 & 0 & -3 \\
-1 & 0 & 1 & 0 \\
0 & 3 & 0 & 1
\end{array}\right]\left\{\left[\begin{array}{c}
0 \\
-x_{1} x_{3}+x_{4} \\
x_{1} x_{2} \\
0
\end{array}\right]+\left[\begin{array}{c}
\left(x_{2}-x_{1}\right) \widehat{a}_{1} \\
x_{1} \widehat{a}_{2}-x_{2} \widehat{a}_{3} \\
-x_{3} \widehat{a}_{4} \\
x_{2} \widehat{a}_{5}-x_{4} \widehat{a}_{6}
\end{array}\right]\right\} \\
& +\left[\begin{array}{cccc}
1 & 0 & 0 & 2 \\
0 & 2 & -3 & 0 \\
0 & 0 & 1 & 1 \\
0 & 3 & 0 & 2
\end{array}\right]\left[\begin{array}{c}
y_{1} \tilde{b}_{1} \\
y_{2} \widetilde{b}_{2} \\
y_{3} \widetilde{b}_{3} \\
0
\end{array}\right]-\left[\begin{array}{cccc}
0 & 1 & 3 & 0 \\
2 & 0 & 0 & -3 \\
-1 & 0 & 1 & 0 \\
0 & 3 & 0 & 1
\end{array}\right]\left[\begin{array}{c}
\left(x_{2}-x_{1}\right) \tilde{a}_{1} \\
x_{1} \widetilde{a}_{2}-x_{2} \widetilde{a}_{3} \\
-x_{3} \tilde{a}_{4} \\
x_{2} \widetilde{a}_{5}-x_{4} \tilde{a}_{6}
\end{array}\right]+\left[\begin{array}{cccc}
1 & 0 & 0 & 2 \\
0 & 2 & -3 & 0 \\
0 & 0 & 1 & 1 \\
0 & 3 & 0 & 2
\end{array}\right]\left[\begin{array}{c}
u_{1} \\
u_{2} \\
u_{3} \\
u_{4}
\end{array}\right]
\end{aligned}
$$

or

$$
\begin{aligned}
& \dot{e}=\left[\begin{array}{c}
-y_{2} y_{3}+y_{4}+y_{1} \widehat{b}_{1}-2 y_{2} \\
-2 y_{1} y_{3}-2 y_{2} \widehat{b}_{2}-3 y_{1} y_{2}-3 y_{1} y_{4}+3 y_{3} \widehat{b}_{3} \\
y_{1} y_{2}+y_{1} y_{4}-y_{3} \widehat{b}_{3}-y_{2} \\
-3 y_{1} y_{3}-3 y_{2} \widehat{b}_{2}-2 y_{2}
\end{array}\right]-\left[\begin{array}{c}
x_{1} \widehat{a}_{2}-x_{2} \widehat{a}_{3}-x_{1} x_{3}+x_{4}+3 x_{2} \widehat{a}_{5}+3 x_{4} \widehat{a}_{6} \\
2\left(x_{2}-x_{1}\right) \widehat{a}_{1}+3 x_{2} \widehat{a}_{5}+x_{4} \widehat{a}_{6} \\
-\left(x_{2}-x_{1}\right) \widehat{a}_{1}-x_{3} \widehat{a}_{4}+x_{1} x_{2} \\
3 x_{1} \widehat{a}_{2}-3 x_{2} \widehat{a}_{3}-3 x_{1} x_{3}+3 x_{4}-x_{2} \widehat{a}_{5}-x_{4} \widehat{a}_{6}
\end{array}\right] \\
& +\left[\begin{array}{c}
y_{1} \tilde{b}_{1} \\
-2 y_{2} \tilde{b}_{2}+2 y_{3} \tilde{b}_{3} \\
-y_{3} \tilde{b}_{3} \\
-3 y_{2} \tilde{b}_{2}
\end{array}\right]-\left[\begin{array}{c}
x_{1} \tilde{a}_{2}-x_{2} \tilde{a}_{3}-3 x_{3} \tilde{a}_{4} \\
2\left(x_{2}-x_{1}\right) \tilde{a}_{1}+3 x_{2} \tilde{a}_{5}+3 x_{4} \tilde{a}_{6} \\
-\left(x_{2}-x_{1}\right) \tilde{a}_{1}-x_{3} \tilde{a}_{4} \\
-3 x_{3} \tilde{a}_{4}-x_{2} \tilde{a}_{5}-x_{4} \tilde{a}_{6}
\end{array}\right]+\left[\begin{array}{cccc}
1 & 0 & 0 & 2 \\
0 & 2 & -3 & 0 \\
0 & 0 & 1 & 1 \\
0 & 3 & 0 & 2
\end{array}\right]\left[\begin{array}{l}
u_{1} \\
u_{2} \\
u_{3} \\
u_{4}
\end{array}\right] . \\
& \dot{e}=\left[\begin{array}{c}
-y_{2} y_{3}+y_{4}+y_{1} \widehat{b}_{1}-2 y_{2}-x_{1} \widehat{a}_{2}+x_{2} \widehat{a}_{3}+x_{1} x_{3}-x_{4}-3 x_{2} \widehat{a}_{5}-3 x_{4} \widehat{a}_{6} \\
-2 y_{1} y_{3}-2 y_{2} \widehat{b}_{2}-3 y_{1} y_{2}-3 y_{1} y_{4}+3 y_{3} \widehat{b}_{3}-2\left(x_{2}-x_{1}\right) \widehat{a}_{1}-3 x_{2} \widehat{a}_{5}-x_{4} \widehat{a}_{6} \\
y_{1} y_{2}+y_{1} y_{4}-y_{3} \widehat{b}_{3}-y_{2}+\left(x_{2}-x_{1}\right) \widehat{a}_{1}+x_{3} \widehat{a}_{4}-x_{1} x_{2} \\
-3 y_{1} y_{3}-3 y_{2} \widehat{b}_{2}-2 y_{2}-3 x_{1} \widehat{a}_{2}+3 x_{2} \widehat{a}_{3}+3 x_{1} x_{3}-3 x_{4}+x_{2} \widehat{a}_{5}+x_{4} \widehat{a}_{6}
\end{array}\right] \\
& +\left[\begin{array}{c}
y_{1} \tilde{b}_{1}-x_{1} \tilde{a}_{2}+x_{2} \tilde{a}_{3}+-3 x_{3} \tilde{a}_{4} \\
-2 y_{2} \tilde{b}_{2}+2 y_{3} \tilde{b}_{3}-2\left(x_{2}-x_{1}\right) \tilde{a}_{1}-3 x_{2} \tilde{a}_{5}-3 x_{4} \tilde{a}_{6} \\
-y_{3} \tilde{b}_{3}+\left(x_{3}-x_{1}\right) \tilde{a}_{1}+x_{3} \tilde{a}_{4} \\
-3 y_{2} \tilde{b}_{2}+3 x_{3} \tilde{a}_{4}+x_{2} \tilde{a}_{5}+x_{4} \tilde{a}_{6}
\end{array}\right]+\left[\begin{array}{cccc}
1 & 0 & 0 & 2 \\
0 & 2 & -3 & 0 \\
0 & 0 & 1 & 1 \\
0 & 3 & 0 & 2
\end{array}\right]\left[\begin{array}{l}
u_{1} \\
u_{2} \\
u_{3} \\
u_{4}
\end{array}\right] .
\end{aligned}
$$

By choosing

$$
\left[\begin{array}{l}
u_{1} \\
u_{2} \\
u_{3} \\
u_{4}
\end{array}\right]=\left[\begin{array}{cccc}
1 & 0 & 0 & 2 \\
0 & 2 & -3 & 0 \\
0 & 0 & 1 & 1 \\
0 & 3 & 0 & 2
\end{array}\right]^{-1}\left\{\left[\begin{array}{r}
-y_{2} y_{3}+y_{4}+y_{1} \widehat{b}_{1}-2 y_{2}-x_{1} \widehat{a}_{2}+x_{2} \widehat{a}_{3}+x_{1} x_{3}-x_{4}-3 x_{2} \widehat{a}_{5}-3 x_{4} \widehat{a}_{6} \\
-2 y_{1} y_{3}-2 y_{2} \widehat{b}_{2}-3 y_{1} y_{2}-3 y_{1} y_{4}+3 y_{3} \widehat{b}_{3}-2\left(x_{2}-x_{1}\right) \widehat{a}_{1}-3 x_{2} \widehat{a}_{5}-x_{4} \widehat{a}_{6} \\
y_{1} y_{2}+y_{1} y_{4}-y_{3} \widehat{b}_{3}-y_{2}+\left(x_{2}-x_{1}\right) \widehat{a}_{1}+x_{3} \widehat{a}_{4}-x_{1} x_{2} \\
-3 y_{1} y_{3}-3 y_{2} \widehat{b}_{2}-2 y_{2}-3 x_{1} \widehat{a}_{2}+3 x_{2} \widehat{a}_{3}+3 x_{1} x_{3}-3 x_{4}+x_{2} \widehat{a}_{5}+x_{4} \widehat{a}_{6}
\end{array}\right]+\left[\begin{array}{c}
e_{2} \\
e_{3} \\
e_{4} \\
v
\end{array}\right]\right\}
$$


the error system becomes

$$
\dot{e}=\left[\begin{array}{c}
e_{2} \\
e_{3} \\
e_{4} \\
v
\end{array}\right]+\left[\begin{array}{c}
y_{1} \tilde{b}_{1}-x_{1} \tilde{a}_{2}+x_{2} \tilde{a}_{3}+-3 x_{3} \tilde{a}_{4} \\
-2 y_{2} \tilde{b}_{2}+3 y_{3} \widetilde{b}_{3}-2\left(x_{2}-x_{1}\right) \tilde{a}_{1}-3 x_{2} \tilde{a}_{5}-3 x_{4} \tilde{a}_{6} \\
-y_{3} \widetilde{b}_{3}+\left(x_{2}-x_{1}\right) \tilde{a}_{1}+x_{3} \tilde{a}_{4} \\
-3 y_{2} \tilde{b}_{2}+3 x_{3} \tilde{a}_{4}+x_{2} \tilde{a}_{5}+x_{4} \tilde{a}_{6}
\end{array}\right],
$$

or

$$
\begin{aligned}
& \dot{e}_{1}=e_{2}+y_{1} \widetilde{b}_{1}-x_{1} \tilde{a}_{2}+x_{2} \widetilde{a}_{3}+-3 x_{3} \widetilde{a}_{4}, \\
& \dot{e}_{2}=e_{3}-2 y_{2} \tilde{b}_{2}+3 y_{3} \tilde{b}_{3}-2\left(x_{2}-x_{1}\right) \tilde{a}_{1}-3 x_{2} \tilde{a}_{5}-3 x_{4} \tilde{a}_{6} \text {, } \\
& \dot{e}_{3}=e_{4}-y_{3} \widetilde{b}_{3}+\left(x_{2}-x_{1}\right) \tilde{a}_{1}+x_{3} \widetilde{a}_{4} \text {, } \\
& \dot{e}_{4}=v-3 y_{2} \widetilde{b}_{2}+3 x_{3} \tilde{a}_{4}+x_{2} \tilde{a}_{5}+x_{4} \tilde{a}_{6} \text {. }
\end{aligned}
$$

To engage the ISMC, adopt the nominal system for (34) as

$$
\begin{aligned}
& \dot{e}_{2}=e_{3}, \\
& \dot{e}_{1}=e_{2}, \\
& \dot{e}_{3}=e_{4}, \\
& \dot{e}_{4}=v_{0} .
\end{aligned}
$$

The sliding surface for the aforementioned nominal system (35) is

$$
\sigma_{0}=\left(1+\frac{d}{\mathrm{~d} t}\right)^{3} e_{1}=e_{1}+3 e_{2}+3 e_{3}+e_{4}
$$

Then,

$$
\dot{\sigma}_{0}=\dot{e}_{1}+3 \dot{e}_{2}+3 \dot{e}_{3}+\dot{e}_{4}=e_{2}+3 e_{3}+3 e_{4}+v_{0} .
$$

By choosing $\nu_{0}=-e_{2}-3 e_{3}-3 e_{4}-k \sigma_{0}, k>0$, we have $\dot{\sigma}_{0}=-k \sigma_{0}$, resulting in the system displayed in (35) is asymptotically stable.

Now, choose the sliding surface for system (34) as

$$
\sigma=\sigma_{0}+z
$$

where the integral term is shown by $z$. To avert the reaching phase, designate $z(0)$ so that $\sigma(0) \longrightarrow 0$. Choose $v=v_{0}+v_{s}$, where $v_{0}$ represents the nominal input and the compensator term is named as $\nu_{s}$. Then,

$$
\begin{aligned}
\dot{\sigma}= & \dot{e}_{1}+3 \dot{e}_{2}+3 \dot{e}_{3}+\dot{e}_{4}+\dot{z}, \\
=e_{2} & +y_{1} \widetilde{b}_{1}-x_{1} \widetilde{a}_{2}+x_{2} \widetilde{a}_{3}+-3 x_{3} \widetilde{a}_{4}+3 e_{3}-6 y_{2} \widetilde{b}_{2}+9 y_{3} \widetilde{b}_{3}-6\left(x_{2}-x_{1}\right) \widetilde{a}_{1}-9 x_{2} \tilde{a}_{5}-9 x_{4} \tilde{a}_{6} \\
& +3 e_{4}-3 y_{3} \tilde{b}_{3}+3\left(x_{2}-x_{1}\right) \tilde{a}_{1}+3 x_{3} \widetilde{a}_{4}-3 y_{2} \tilde{b}_{2}+3 x_{3} \tilde{a}_{4}+x_{2} \tilde{a}_{5}+x_{4} \tilde{a}_{6}+v_{0}+v_{s}+\dot{z} .
\end{aligned}
$$

If we choose a Lyapunov function

$$
V=\frac{1}{2} \sigma^{2}+\frac{1}{2}\left(\widetilde{a}_{1}^{2}+\widetilde{a}_{2}^{2}+\widetilde{a}_{3}^{2}+\widetilde{a}_{4}^{2}+\widetilde{b}_{1}^{2}+\widetilde{b}_{2}^{2}+\widetilde{b}_{3}^{2}\right),
$$

and then if adaptive laws are chosen as

$$
\begin{aligned}
& \dot{z}=-e_{2}-3 e_{3}-3 e_{4}-v_{0}, \quad v_{s}=-k \sigma_{0}, \\
& \dot{\tilde{a}}_{1}=3 \sigma\left(x_{2}-x_{1}\right)-k_{1} \widetilde{a}_{1}, \quad \dot{\hat{a}}_{1}=\dot{\tilde{a}}_{1}, \\
& \dot{\tilde{a}}_{2}=\sigma x_{1}-k_{2} \tilde{a}_{2}, \quad \dot{\hat{a}}_{2}=\dot{\tilde{a}}_{2}, \\
& \dot{\tilde{a}}_{3}=-\sigma x_{2}-k_{3} \widetilde{a}_{3}, \quad \dot{\hat{a}}_{3}=\dot{\tilde{a}}_{3}, \\
& \dot{\tilde{a}}_{4}=-9 \sigma x_{3}-k_{4} \tilde{a}_{4}, \quad \dot{\hat{a}}_{4}=\dot{\tilde{a}}_{4},
\end{aligned}
$$

$$
\begin{array}{ll}
\dot{\tilde{a}}_{5}=-8 \sigma x_{2}-k_{5} \tilde{a}_{5}, & \dot{\hat{a}}_{5}=\dot{\tilde{a}}_{5}, \\
\dot{\tilde{a}}_{6}=-8 \sigma x_{4}-k_{6} \widetilde{a}_{6}, & \dot{\hat{a}}_{6}=\dot{\tilde{a}}_{6}, \\
\dot{\widetilde{b}}_{1}=-\sigma y_{1}-k_{7} \widetilde{b}_{1}, & \dot{\hat{b}}_{1}=\dot{\tilde{b}}_{1}, \\
\dot{\widetilde{b}}_{2}=-9 \sigma y_{2}-k_{8} \tilde{b}_{2}, & \dot{\hat{b}}_{2}=\dot{\tilde{b}}_{2}, \\
\dot{\widetilde{b}}_{3}=-6 \sigma y_{3}-k_{9} \widetilde{b}_{3}, & \dot{\hat{b}}_{3}=\dot{\vec{b}}_{3},
\end{array}
$$

then $\dot{V}<0$.

It can be observed from Figure 5 that the error converges to zero very rapidly. The initial value of the error must depend on the initial condition of master and slave states. Since 


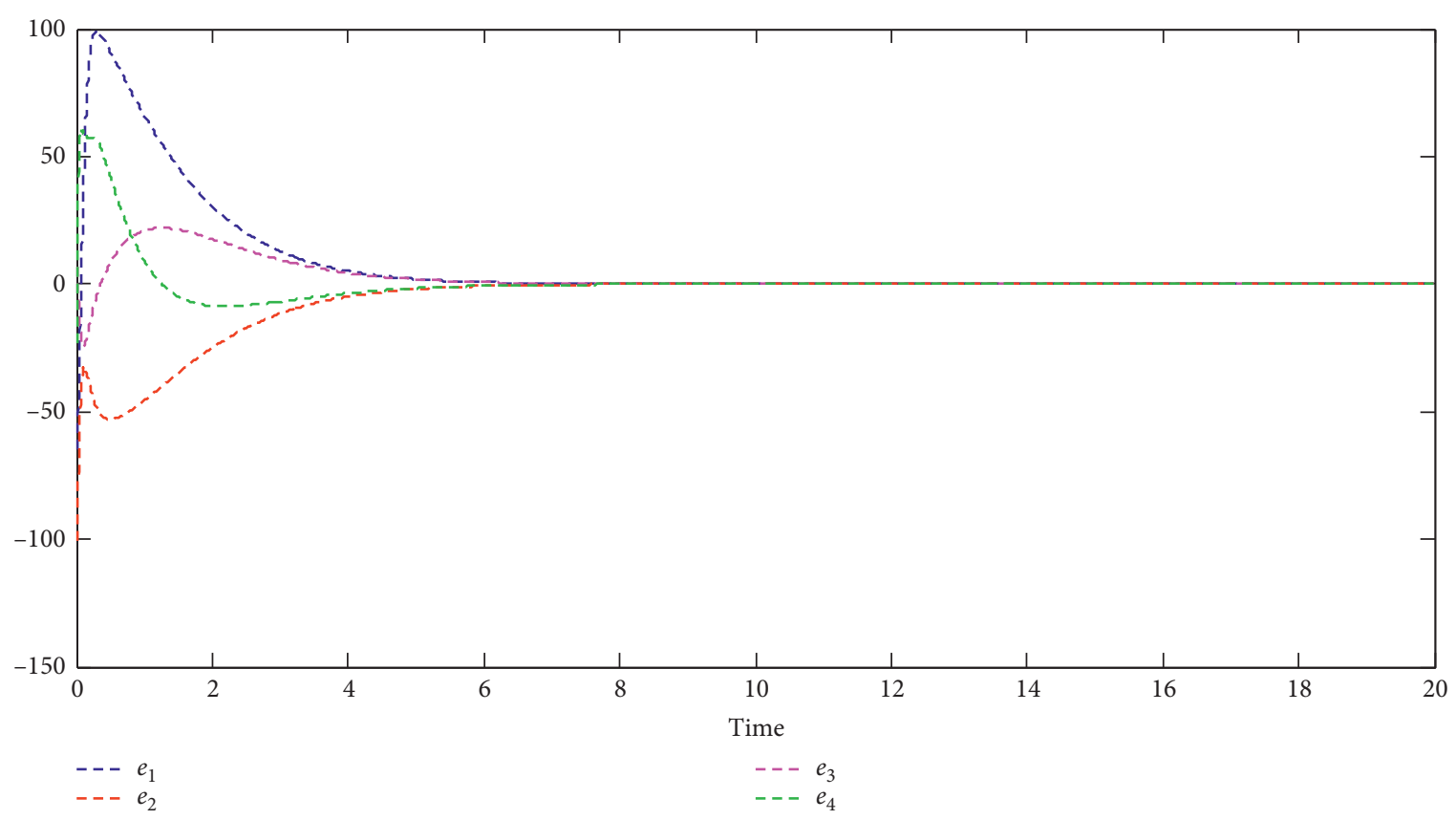

Figure 5: Synchronization errors.

$$
\begin{aligned}
& \dot{V}=\sigma \dot{\sigma}+\widetilde{a}_{1} \dot{\tilde{a}}_{1}+\tilde{a}_{2} \dot{\tilde{a}}_{2}+\tilde{a}_{3} \dot{\tilde{a}}_{3}+\widetilde{a}_{4} \dot{\tilde{a}}_{4}+\widetilde{a}_{5} \dot{\tilde{a}}_{5}+\tilde{a}_{6} \dot{\tilde{a}}_{6}+\widetilde{b}_{1} \dot{\vec{b}}_{1}+\widetilde{b}_{2} \dot{\tilde{b}}_{2}+\widetilde{b}_{3} \dot{\tilde{b}}_{3} \\
& \dot{V}=\sigma\left\{e_{2}+y_{1} \widetilde{b}_{1}-x_{1} \tilde{a}_{2}+x_{2} \tilde{a}_{3}+-3 x_{3} \tilde{a}_{4}+3 e_{3}-6 y_{2} \widetilde{b}_{2}+9 y_{3} \widetilde{b}_{3}-6\left(x_{2}-x_{1}\right) \tilde{a}_{1}\right. \\
& -9 x_{2} \tilde{a}_{5}-9 x_{4} \tilde{a}_{6}+3 e_{4}-3 y_{3} \widetilde{b}_{3}+3\left(x_{2}-x_{1}\right) \tilde{a}_{1}+3 x_{3} \tilde{a}_{4}-3 y_{2} \widetilde{b}_{2}+3 x_{3} \tilde{a}_{4} \\
& \left.+x_{2} \tilde{a}_{5}+x_{4} \tilde{a}_{6}+v_{0}+v_{s}+\dot{z}\right\}+\tilde{a}_{1} \dot{\tilde{a}}_{1}+\tilde{a}_{2} \dot{\tilde{a}}_{2}+\tilde{a}_{3} \dot{\tilde{a}}_{3}+\tilde{a}_{4} \dot{\tilde{a}}_{4}+\tilde{a}_{5} \dot{\tilde{a}}_{5}+\tilde{a}_{6} \dot{\tilde{a}}_{6}+\widetilde{b}_{1} \dot{\vec{b}}_{1}+\widetilde{b}_{2} \dot{\tilde{b}}_{2}+\widetilde{b}_{3} \dot{\tilde{b}}_{3} \\
& =\sigma\left\{e_{2}+3 e_{3}+3 e_{4}+v_{0}+v_{s}+\dot{z}\right\}+\tilde{a}_{1}\left\{\dot{\tilde{a}}_{1}-3 \sigma\left(x_{2}-x_{1}\right)\right\}+\tilde{a}_{2}\left\{\dot{\tilde{a}}_{2}-\sigma x_{1}\right\} \\
& +\tilde{a}_{3}\left\{\dot{\tilde{a}}_{3}+\sigma x_{2}\right\}+\tilde{a}_{4}\left\{\dot{\tilde{a}}_{4}+9 \sigma x_{3}\right\}+\tilde{a}_{5}\left\{\dot{\tilde{a}}_{5}-8 \sigma x_{2}\right\}+\tilde{a}_{6}\left\{\dot{\tilde{a}}_{6}+8 \sigma x_{4}\right\} \\
& +\widetilde{b}_{1}\left\{\dot{\vec{b}}_{1}+\sigma y_{1}\right\}+\widetilde{b}_{2}\left\{\dot{\vec{b}}_{2}-9 \sigma y_{2}\right\}+\widetilde{b}_{3}\left\{\dot{\vec{b}}_{3}+6 \sigma y_{3}\right\} \text {, } \\
& \dot{z}=-e_{2}-3 e_{3}-3 e_{4}-v_{0}, \quad v_{s}=-k \sigma, \\
& \dot{\tilde{a}}_{1}=3 \sigma\left(x_{2}-x_{1}\right)-k_{1} \widetilde{a}_{1}, \quad \dot{\hat{a}}_{1}=\dot{\tilde{a}}_{1}, \\
& \dot{\tilde{a}}_{2}=\sigma x_{1}-k_{2} \tilde{a}_{2}, \quad \dot{\vec{a}}_{2}=\dot{\tilde{a}}_{2}, \\
& \dot{\tilde{a}}_{3}=-\sigma x_{2}-k_{3} \widetilde{a}_{3}, \quad \dot{\hat{a}}_{3}=\dot{\tilde{a}}_{3}, \\
& \dot{\tilde{a}}_{4}=-9 \sigma x_{3}-k_{4} \widetilde{a}_{4}, \quad \dot{\hat{a}}_{4}=\dot{\tilde{a}}_{4}, \\
& \dot{\tilde{a}}_{5}=-8 \sigma x_{2}-k_{5} \widetilde{a}_{5}, \quad \dot{\hat{a}}_{5}=\dot{\tilde{a}}_{5}, \\
& \dot{\tilde{a}}_{6}=-8 \sigma x_{4}-k_{6} \widetilde{a}_{6}, \quad \dot{\hat{a}}_{6}=\dot{\tilde{a}}_{6} \text {, } \\
& \dot{\widetilde{b}}_{1}=-\sigma y_{1}-k_{7} \widetilde{b}_{1}, \quad \dot{\vec{b}}_{1}=\dot{\widetilde{b}}_{1} \text {, } \\
& \dot{\tilde{b}}_{2}=-9 \sigma y_{2}-k_{8} \widetilde{b}_{2}, \quad \dot{\hat{b}}_{2}=\dot{\widetilde{b}}_{2} \text {, } \\
& \dot{\tilde{b}}_{3}=-6 \sigma y_{3}-k_{9} \widetilde{b}_{3}, \quad \dot{\hat{b}}_{3}=\dot{\widetilde{b}}_{3} \text {. }
\end{aligned}
$$




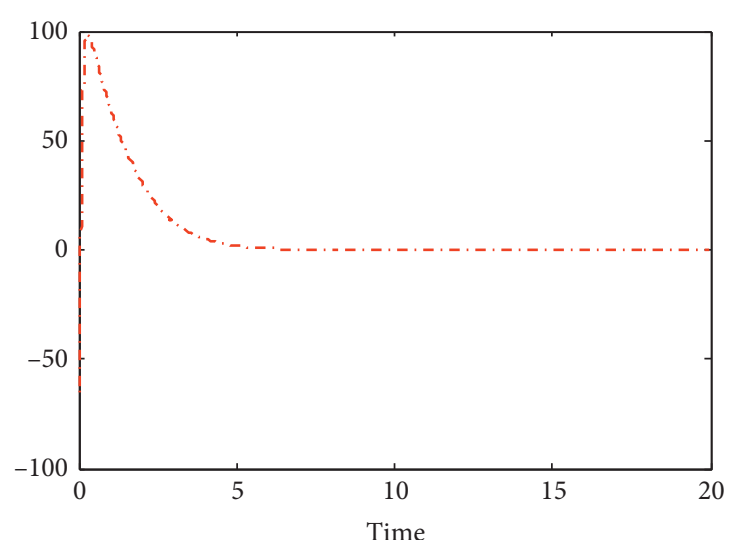

$-\cdot e_{1}=\left(y_{1}+2 y_{4}\right)-\left(x_{2}+3 x_{3}\right)$

(a)

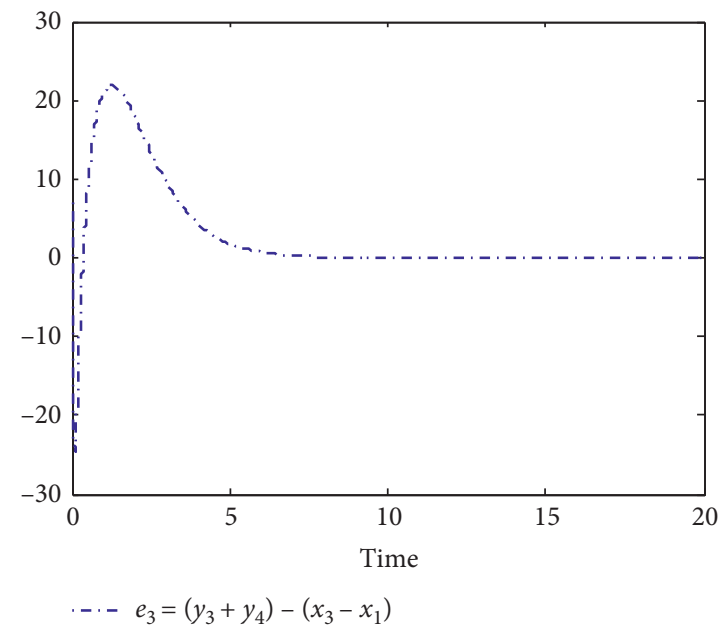

(c)

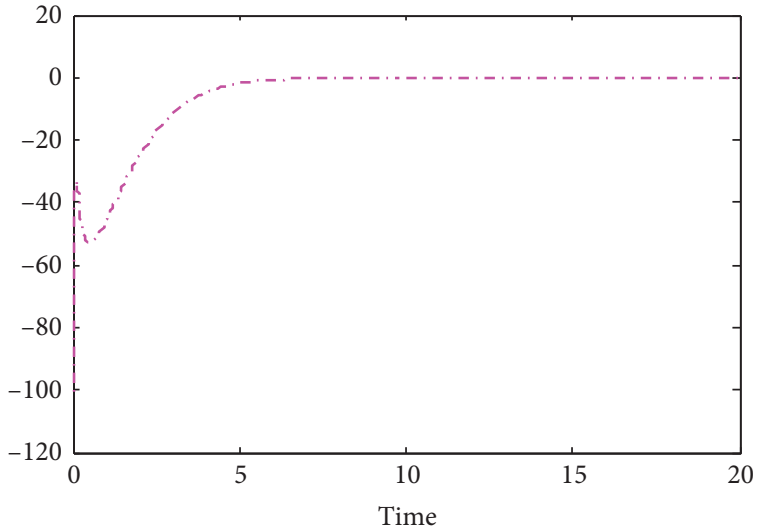

$-e_{2}=\left(2 y_{2}-3 y_{3}\right)-\left(2 x_{1}-3 x_{4}\right)$

(b)

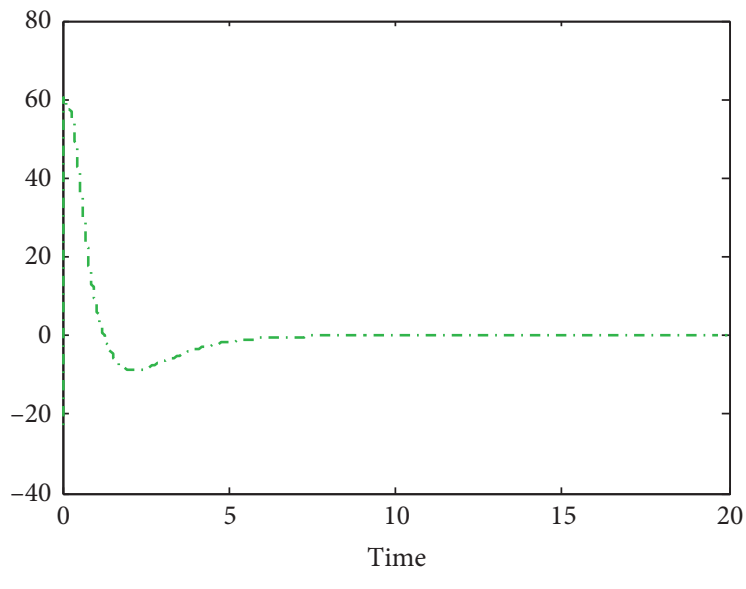

$-e_{4}=\left(3 y_{2}+2 y_{4}\right)-\left(3 x_{2}+x_{4}\right)$

(d)

FIGURE 6: Error convergence towards zero.

We have

$$
\begin{aligned}
\dot{V}= & -k \sigma^{2}-k_{1} \widetilde{a}_{1}^{2}-k_{2} \tilde{a}_{2}^{2}-k_{3} \widetilde{a}_{3}^{2}-k_{4} \widetilde{a}_{4}^{2}-k_{5} \widetilde{a}_{5}^{2} \\
& -k_{6} \widetilde{a}_{6}^{2}-k_{7} \widetilde{b}_{1}^{2}-k_{8} \widetilde{b}_{2}^{2}-k_{9} \widetilde{b}_{3}^{2} .
\end{aligned}
$$

Since $\sigma \longrightarrow 0$, therefore, $e_{1}, e_{2}, e_{3}, e_{4} \longrightarrow 0$.

For simulation, the initial conditions are chosen as $\left(x_{1}(0), x_{2}(0), x_{3}(0), x_{4}(0)\right)=(8.3,10.8,17.4,-11.1)$ and

$$
\left(y_{1}(0), y_{2}(0), y_{3}(0), y_{4}(0)\right)=(-0.2,-0.1,16.9,-0.7) \text {. }
$$

True values of the unknown parameters are chosen as

$$
\begin{aligned}
& a_{1}=12, a_{2}=23, a_{3}=1, a_{4}=2.1, a_{5}=6, \\
& a_{6}=0.2, b_{1}=8, b_{2}=40, b_{3}=14.9 .
\end{aligned}
$$

The corresponding simulation outcomes are shown in Figure 3. It can be observed from Figures 5 and 6 that the errors regarding synchronization rapidly converge to zero. Figures 7 and 8 show the identification processes of unknown parameters, which show that the estimated values tend to their true values adaptively, i.e., $\theta \longrightarrow[122312.160 .2]^{T}$ and $\vartheta \longrightarrow[84014.9]^{T}$. If we may consider equations (39) and (40), adaptive parameters (42) are utilized in the calculation of the sliding surface (in terms of errors). The robustness is the beauty of adaptive parametric stabilization, which may be observed via Figures 5 and 7. The Q-S synchronization is plotted in Figure 8, which indicates response system (22) is synchronized with drive system (21) with respect to the given vector map (26). 


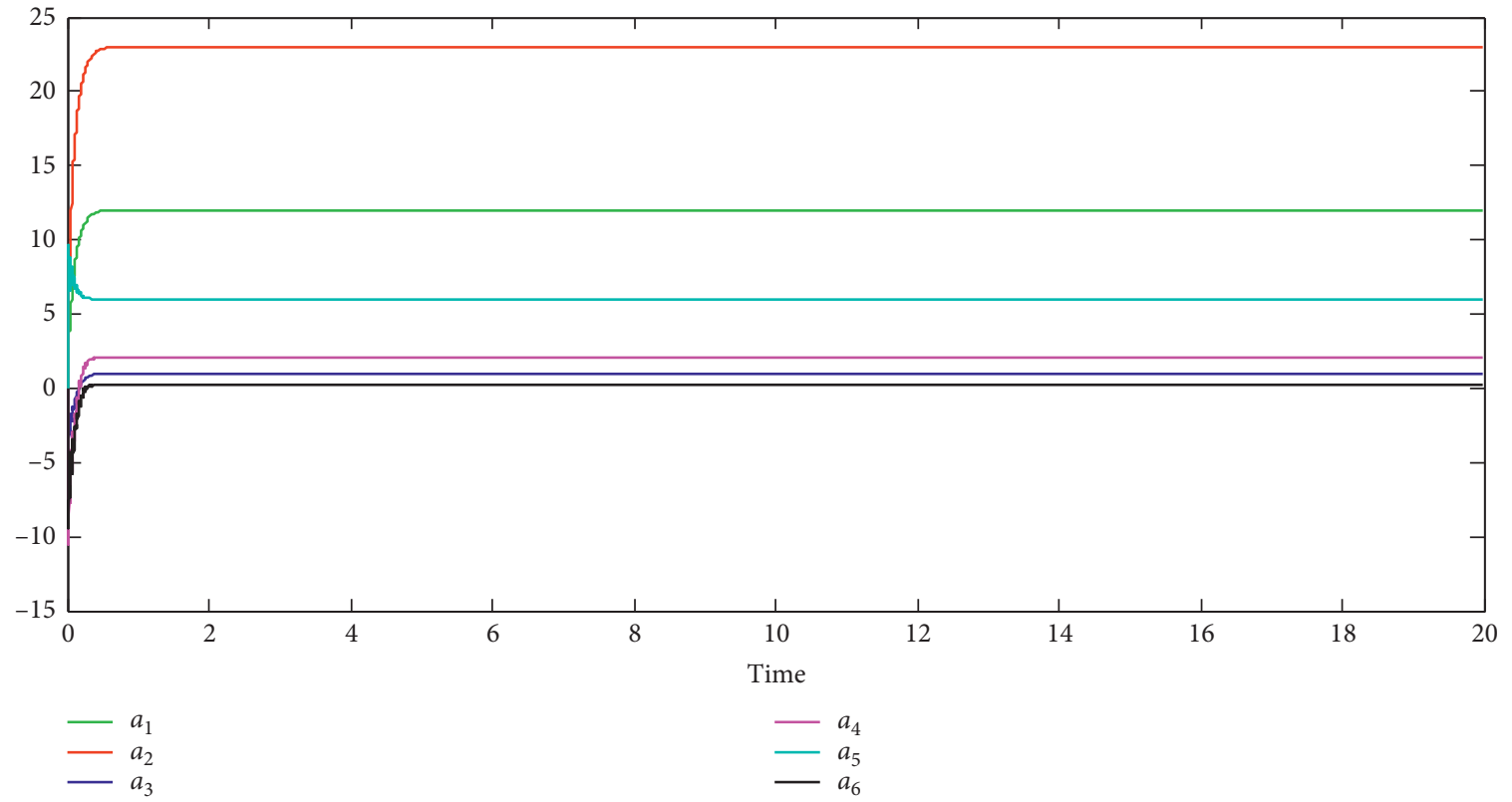

(a)

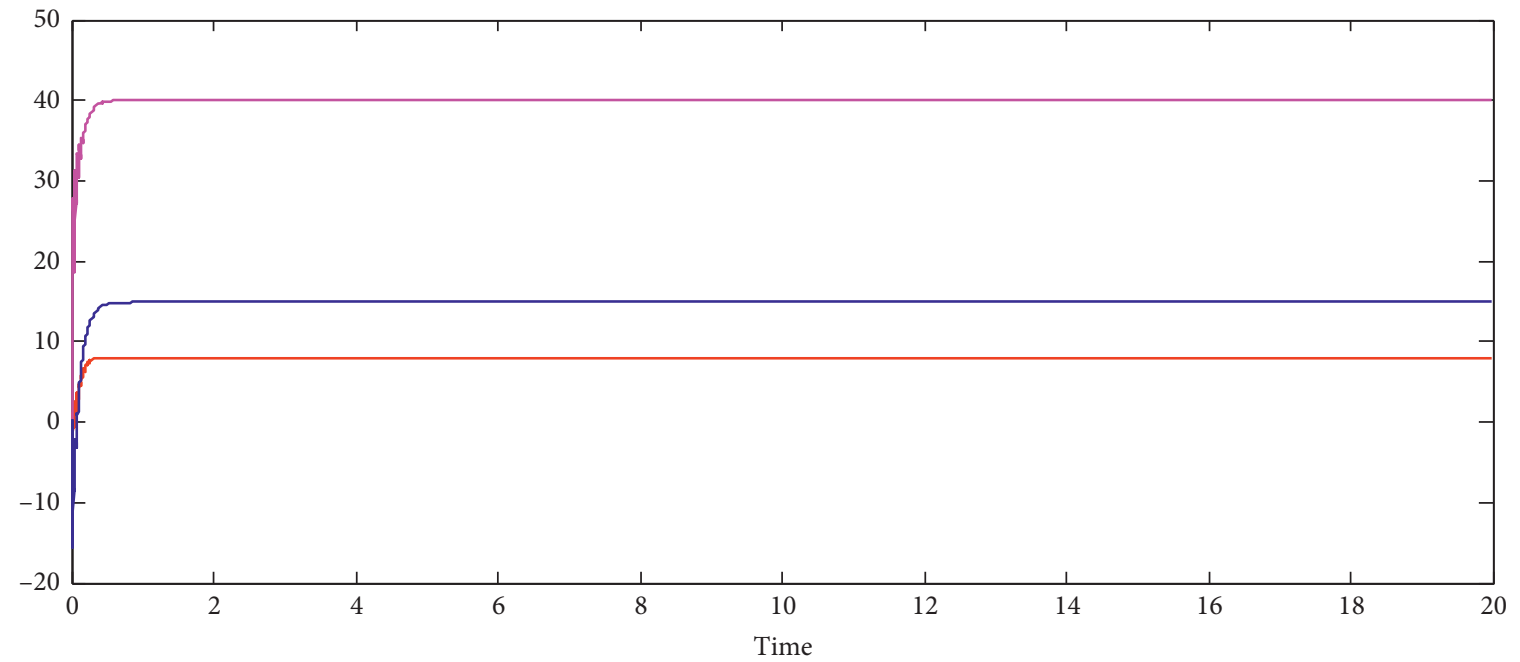

$-b_{1}$
$-b_{2}$
$-b_{3}$

(b)

FIgURE 7: The identification process of unknown parameters.

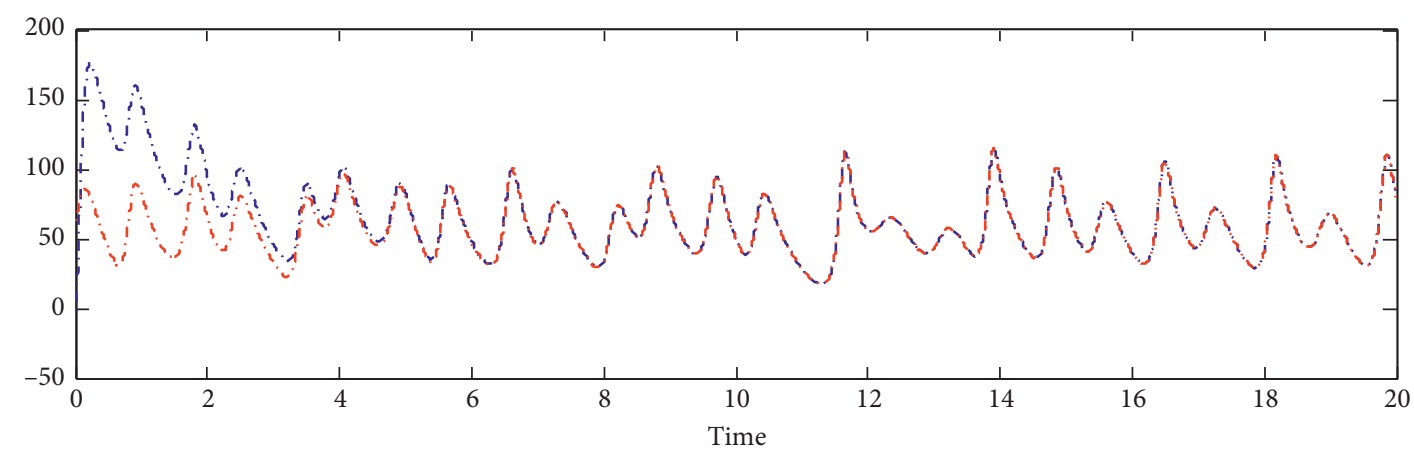

- - $\left(y_{1}+2 y_{4}\right)$

$-\cdot\left(x_{2}+3 x_{3}\right)$

(a)

FIgURE 8: Continued. 


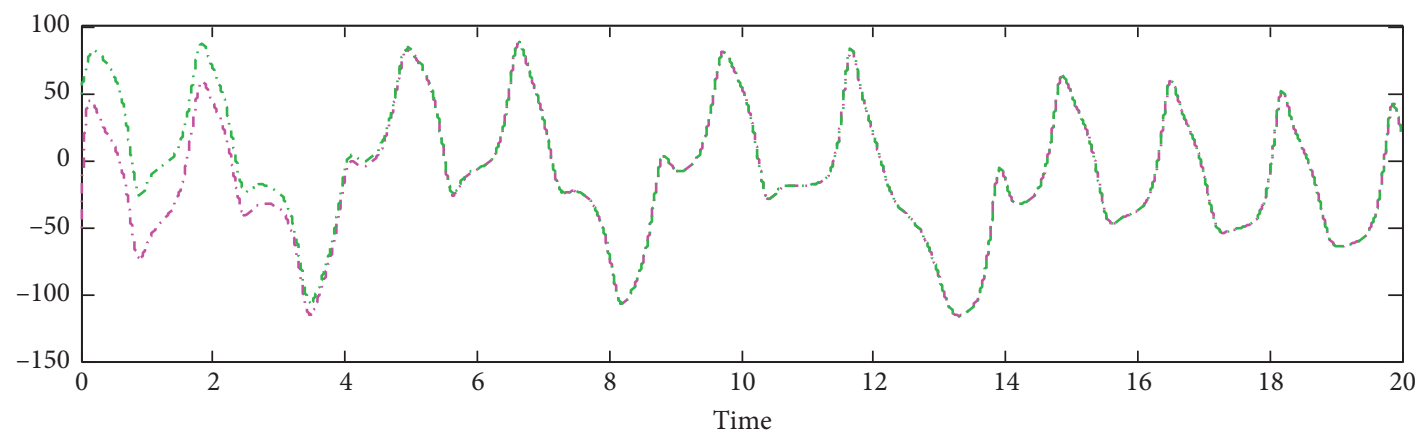

$$
\begin{aligned}
& \text {.. }\left(2 y_{2}-3 y_{3}\right) \\
& \text {-. }\left(2 x_{1}-3 x_{4}\right)
\end{aligned}
$$

(b)

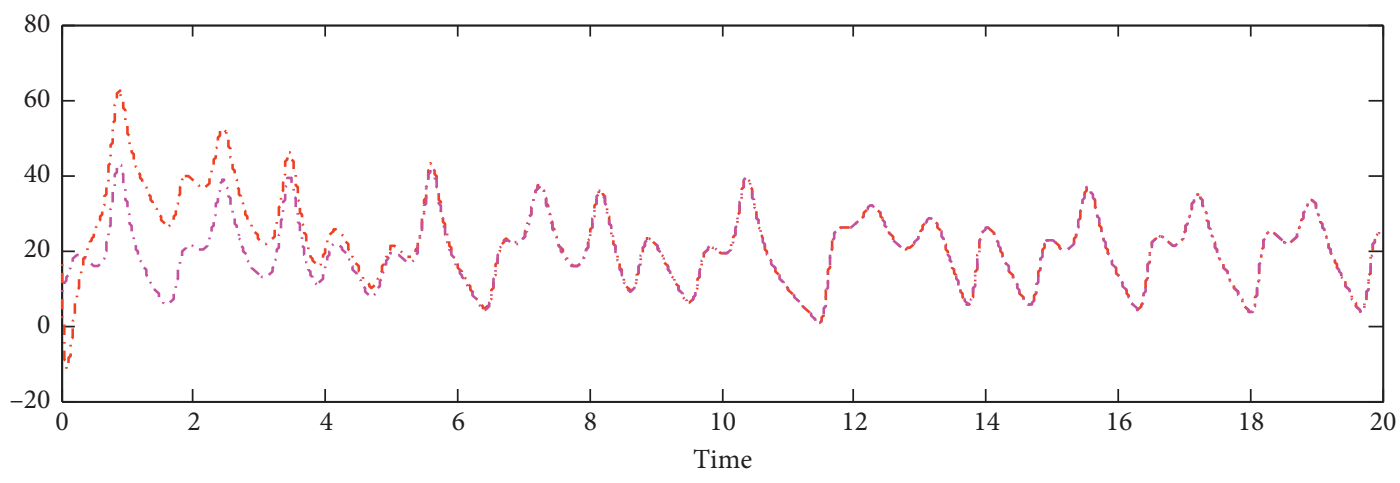

... $\left(y_{3}+y_{4}\right)$

$\cdot-\cdot\left(x_{3}-x_{1}\right)$

(c)

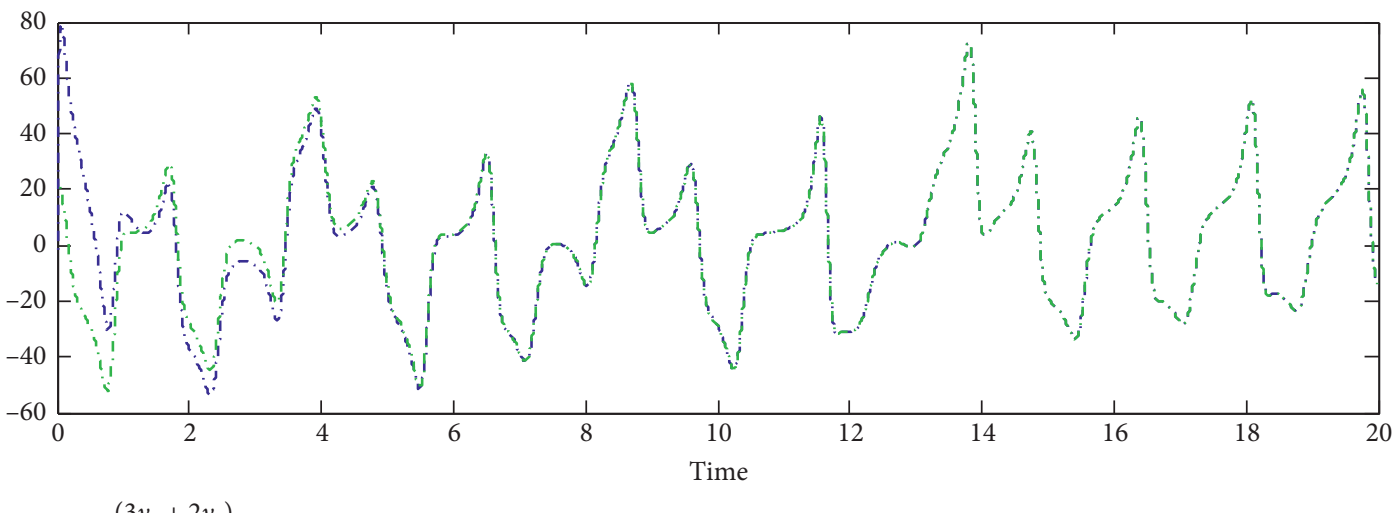

...- $\left(3 y_{2}+2 y_{4}\right)$

-.. $\left(3 x_{2}+x_{4}\right)$

(d)

Figure 8: The Q-S synchronization of the response and drive system.

\section{Conclusion}

A novel Q-S synchronization-based scheme is presented along with its application towards parameter identification and synchronization of two complex nonlinear, nonidentical systems with unknown parameters. The aforementioned strategy relies on adaptive ISMC. The Lyapunov theory is used to prove the stability of the proposed adaptive controller with a parameter estimator. To overcome the high-frequency oscillation (chattering) problem, smooth continuous compensator control is used instead of the traditional discontinuous control. Using Lyapunov stability theory, the compensator controller and the relevant adapted laws are derived and proved via strictly negative Lyapunov function conditions. Simulation results show the effectiveness of the proposed synchronization approach. 


\section{Data Availability}

The data used to support the findings of this study are available from the corresponding author upon request.

\section{Conflicts of Interest}

The authors declare that there are no conflicts of interest regarding the publication of this paper.

\section{References}

[1] O. E. Rossler, "An equation for hyperchaos," Physics Letters A, vol. 71, no. 2-3, pp. 155-157, 1979.

[2] W. Xingyuan and W. Mingjun, "A hyperchaos generated from Lorenz system," Physica A: Statistical Mechanics and its Applications, vol. 387, no. 14, pp. 3751-3758, 2008.

[3] Y. Li, W. K. S. Tang, and G. Chen, "Generating hyperchaos via state feedback control," International Journal of Bifurcation and Chaos, vol. 15, no. 10, pp. 3367-3375, 2005.

[4] A. Chen, J. Lu, J. Lü, and S. Yu, "Generating hyperchaotic Lu attractor via state feedback control," Physica A: Statistical Mechanics and its Applications, vol. 364, pp. 103-110, 2006.

[5] Q. Li and X. S. Yang, "Hyperchaos from two coupled Wienbridge oscillators," International Journal of Circuit Theory and Applications, vol. 36, no. 1, pp. 19-29, 2007.

[6] L. M. Pecora and T. L. Carroll, "Synchronization in chaotic systems," Physical Review Letters, vol. 64, no. 8, pp. 821-824, 1990.

[7] H. Fujisaka and T. Yamada, "Stability theory of synchronized motion in coupled-oscillator systems," Progress of Theoretical Physics, vol. 69, no. 1, pp. 32-47, 1983.

[8] A. S. Pikovsky, "On the interaction of strange attractors," Zeitschrift für Physik B Condensed Matter, vol. 55, no. 2, pp. 149-154, 1984.

[9] F. Zhang, "Lag synchronization of complex Lorenz system with applications to communication," Entropy, vol. 17, no. 7, pp. 4974-4985, 2015.

[10] W. X. Yuan and Z. Hao, "Backstepping-based lag synchronization of a complex permanent magnet synchronous motor system," China Physics B, vol. 22, no. 4, pp. 558-562, 2013.

[11] G.-H. Li, "Inverse lag synchronization in chaotic systems," Chaos, Solitons \& Fractals, vol. 40, no. 3, pp. 1076-1080, 2009.

[12] C. K. Volos, I. M. Kyprianidis, and I. N. Stouboulos, "antiphase and inverse $\pi$-lag synchronization in coupled duffingtype circuitsfing type circuits," International Journal of Bifurcation and Chaos, vol. 21, no. 8, pp. 2357-2368, 2011.

[13] S. Wang, X. Wang, and B. Han, "Complex generalized synchronization and parameter identification of non-identical nonlinear complex systems," PLoS One, vol. 11, no. 3, pp. 1-19, 2016.

[14] H. Afzal, M. R. Mufti, S. U. Din et al., "Modeling and inverse complex generalized synchronization and parameter identification of non-identical nonlinear complex systems using adaptive integral sliding mode control," IEEE Access, vol. 8, pp. 38950-38969, 2020.

[15] R. M. Guerra and J. L. M. Machuca, "Fractional generalized synchronization in a class of nonlinear fractional order systems," Nonlinear Dynamics, vol. 77, pp. 1237-1244, 2014.

[16] X. Chen, J. H. Park, J. Cao, and J. Qiu, "Sliding mode synchronization of multiple chaotic systems with uncertainties and disturbances," Applied Mathematics and Computation, vol. 308, pp. 161-173, 2017.
[17] G. I. Dykman, P. S. Landa, and Y. I. Neymark, "Synchronizing the chaotic oscillations by external force," Chaos, Solitons \& Fractals, vol. 1, no. 4, pp. 339-353, 1991.

[18] F. Q. Dou, J. A. Sun, W. S. Duan, and K. P. Lü, “Anti-synchronization of a new hyperchaotic system," Physica Scripta, vol. 78, no. 1, Article ID 015007, 2008.

[19] R. F. Zhang, D. Y. Chen, and J. G. Yang, "Anti-synchronization for a class of multi-dimensional autonomous and nonautonomous chaotic systems on the basis of the sliding mode with noise," Physica Scripta, vol. 85, no. 6, 2012.

[20] J.-W. Wang and A.-M. Chen, "Partial synchronization in coupled chemical chaotic oscillators," Journal of Computational and Applied Mathematics, vol. 233, no. 8, pp. 18971904, 2010.

[21] D. J. Wagg, "Partial synchronization of nonidentical chaotic systems via adaptive control, with applications to modeling coupled nonlinear systems," International Journal of Bifurcation and Chaos, vol. 12, no. 3, pp. 561-570, 2002.

[22] J. Zhao, "Adaptive Q-S synchronization between coupled chaotic systems with stochastic perturbation and delay," Applied Mathematical Modelling, vol. 36, no. 7, pp. 33123319, 2012.

[23] Z. Y. Yan, "Chaos Q-S synchronization between Rossler system and the new unified chaotic system," Physics Letters A, vol. 334, no. 5-6, pp. 406-412, 2005.

[24] Y. J. Niu and X. Y. Wang, "Projective synchronization of different chaotic systems with nonlinearity inputs," International Journal of Modern Physics B, vol. 26, no. 11, 2012.

[25] D. M. Senejohnny and H. Delavari, "Active sliding observer scheme based fractional chaos synchronization," Communications in Nonlinear Science and Numerical Simulation, vol. 17, no. 11, pp. 4373-4383, 2012.

[26] H. Delavari and M. Mohadeszadeh, "Robust finite-time synchronization of non-identical fractional-order hyperchaotic systems and its application in secure communication," IEEE/CAA Journal of Automatica Sinica, vol. 6, no. 1, pp. 228-235, 2019.

[27] H. U. Voss, "Anticipating chaotic synchronization," Physical Review E, vol. 61, no. 5, pp. 5115-5119, 2000.

[28] M. R. Mufti, H. Afzal, F. U. Rehman, Q. R. Butt, and M. I. Qureshi, "Synchronization and antisynchronization between two non-identical chua oscillators via sliding mode control," IEEE Access, vol. 6, pp. 45270-45280, 2018.

[29] S. U. Din, Q. Khan, F. U. Rehman, and R. Akmeliawati, "Robust control of underactuated systems: higher order integral sliding mode approach," Mathematical Problems in Engineering, vol. 2016, Article ID 5641478, 11 pages, 2016.

[30] S. U. Din, Q. Khan, F.-U. Rehman, and R. Akmeliawanti, "A comparative experimental study of robust sliding mode control strategies for underactuated systems," IEEE Access, vol. 5, pp. 10068-10080, 2017.

[31] H. Delavari, H. Heydarinejad, and D. Baleanu, "Adaptive fractional-order blood glucose regulator based on high-order sliding mode observer," IET Systems Biology, vol. 13, no. 2, pp. 43-54, 2019.

[32] H. Lee and V. I. Utkin, "Chattering suppression methods in sliding mode control systems," Annual Reviews in Control, vol. 31, no. 2, pp. 179-188, 2007.

[33] H. Delavari, D. M. Senejohnny, and D. Baleanu, "Sliding observer for synchronization of fractional order chaotic systems with mismatched parameter," Central European Journal of Physics, vol. 10, no. 5, pp. 1095-1101, 2012.

[34] Y. Li, X. Liu, G. Chen, and X. Liao, "A new hyperchaotic Lorenz-type system: generation, analysis, and 
implementation," International Journal of Circuit Theory and Applications, vol. 39, no. 8, pp. 865-879, 2012.

[35] S. Dadras, H. R. Momeni, G. Qi, and Z.-l. Wang, "Four-wing hyperchaotic attractor generated from a new $4 \mathrm{D}$ system with one equilibrium and its fractional-order form," Nonlinear Dynamics, vol. 67, no. 2, pp. 1161-1173, 2012.

[36] S. U. Din, F. U. Rehman, and Q. Khan, "Synchronization of 4D hyperchaotic Rikitake dynamo system along with unknown parameters via adaptive sliding mode," Control Engineering and Applied Informatics, vol. 20, pp. 108-118, 2018. 